\title{
Modelling the effect of acoustic waves on the thermodynamics and kinetics of phase transformation in a solution: Including mass transportation
}

Cite as: J. Chem. Phys. 148, 024102 (2018); https://doi.org/10.1063/1.5003021

Submitted: 01 September 2017 . Accepted: 20 December 2017 . Published Online: 08 January 2018

S. R. Haqshenas, I. J. Ford (D), and N. Saffari (D)

\section{ARTICLES YOU MAY BE INTERESTED IN}

Perspective: Structural fluctuation of protein and Anfinsen's thermodynamic hypothesis The Journal of Chemical Physics 148, 020901 (2018); https://doi.org/10.1063/1.5013104

Modelling the effect of acoustic waves on nucleation

The Journal of Chemical Physics 145, 024315 (2016); https://doi.org/10.1063/1.4955202

Molecular dynamics based enhanced sampling of collective variables with very large time steps

The Journal of Chemical Physics 148, 024106 (2018); https://doi.org/10.1063/1.4999447

PHYSICS TODAY WHITEPAPERS
ADVANCED LIGHT CURE ADHESIVES

Take a closer look at what these

environmentally friendly adhesive systems can do
READ NOW

PRESENTED BY Q MASTERBOND: 


\title{
Modelling the effect of acoustic waves on the thermodynamics and kinetics of phase transformation in a solution: Including mass transportation
}

\author{
S. R. Haqshenas, ${ }^{1, a)}$ I. J. Ford, ${ }^{2, b)}$ and N. Saffari ${ }^{1, c)}$ \\ ${ }^{1}$ Department of Mechanical Engineering, University College London, Gower Street, \\ London WC1E 7JE, United Kingdom \\ ${ }^{2}$ Department of Physics and Astronomy, University College London, Gower Street, \\ London WC1E 6BT, United Kingdom
}

(Received 1 September 2017; accepted 20 December 2017; published online 8 January 2018)

\begin{abstract}
Effects of acoustic waves on a phase transformation in a metastable phase were investigated in our previous work [S. R. Haqshenas, I. J. Ford, and N. Saffari, "Modelling the effect of acoustic waves on nucleation," J. Chem. Phys. 145, 024315 (2016)]. We developed a non-equimolar dividing surface cluster model and employed it to determine the thermodynamics and kinetics of crystallisation induced by an acoustic field in a mass-conserved system. In the present work, we developed a master equation based on a hybrid Szilard-Fokker-Planck model, which accounts for mass transportation due to acoustic waves. This model can determine the kinetics of nucleation and the early stage of growth of clusters including the Ostwald ripening phenomenon. It was solved numerically to calculate the kinetics of an isothermal sonocrystallisation process in a system with mass transportation. The simulation results show that the effect of mass transportation for different excitations depends on the waveform as well as the imposed boundary conditions and tends to be noticeable in the case of shock waves. The derivations are generic and can be used with any acoustic source and waveform. Published by AIP Publishing. https://doi.org/10.1063/1.5003021
\end{abstract}

\section{INTRODUCTION}

Acoustic waves propagate in a fluid through consecutive compression and rarefaction cycles. The rarefaction cycle can nucleate bubbles or induce a liquid-gas transition ${ }^{1-3}$ called acoustic cavitation. In the case of a solid new phase (either a liquid-solid transition or crystallisation from a solution), the process of phase transition is called sonocrystallisation which has been used for the production and purification of solid particles in various industries, e.g., pharmaceutical and food. ${ }^{4}$ During propagation of acoustic waves in the fluid, acoustic energy may be partially converted into thermal energy through different mechanisms, e.g., viscous losses and/or relaxational losses. $^{5-8}$ Therefore, depending on the excitation period, the temperature may vary at some spatial locations. Furthermore, acoustic waves may distort due to the nonlinearity in the equation of state of the fluid, and shock waves can be formed. ${ }^{8}$ The temperature, flow, and pressure fields at each spatial location in the liquid old phase are comprised of the direct field from the acoustic source and the indirect field generated by acoustic cavitation when this phenomenon occurs. The latter is a localised effect which is limited to the vicinity of bubbles. This effect can however be substantial if inertial cavitation occurs. ${ }^{9-11}$ These varying temperature, flow, and pressure fields imply that a phase transition in an acoustic

\footnotetext{
a)seyyed.haqshenas.12@ucl.ac.uk

b)i.ford@ucl.ac.uk

c)n.saffari@ucl.ac.uk
}

field is unsteady and the use of conventional equations based on the assumption of a stationary process is not justified in general. This motivates the need to treat sonocrystallisation as an unsteady process which shapes our vision for modeling.

We recently developed a new model that can describe the thermodynamics of phase transition for small clusters. ${ }^{12}$ This is important as the size of the critical cluster reduces with an increase in the intensity of the acoustic field. Describing the kinetics of cluster formation by the cluster dynamics, the aggregative and non-aggregative mechanisms are the two main governing mechanisms which determine the cluster distribution over time. ${ }^{13}$ Aggregative mechanisms include nucleation, growth, and ageing, which give rise to the flux of the cluster concentration along the size axis. The non-aggregative mechanism accounts for the change in the concentration of clusters in a system driven by mass flux along the space parameter axis, e.g., due to forced diffusion in an acoustic field. Such a system is called a non-mass conserved system here. The thermodynamics and kinetics of phase transformation in a massconserved system (overlooking the non-aggregative mechanism) exposed to an acoustic field were studied in the preceding work. ${ }^{12}$ The objective of the present paper is to cast a kinetic model, both in discrete and continuous forms, which accounts for the effects of wave propagation on both aggregative and non-aggregative mechanisms of cluster dynamics.

The kinetics of cluster formation in a non-mass conserved system has been studied in different applications, namely: the theory of thin film condensation ${ }^{14-20}$ and phase transition and precipitation in flows. ${ }^{21-27}$ The convection-diffusion equation is typically used to determine mass transportation 
in accord with hydrodynamics. This is incorporated into a kinetic equation in order to compute the kinetics of nucleation or reaction in an open system. ${ }^{28-30}$ The effect of hydrodynamics on the molecular diffusion can be accounted for by using the generic Fick's equation which entails the effect of pressure gradient, temperature gradient, and external forces on the flux of solute molecules in a mixture. ${ }^{16,31,32}$ A simplified form of the convection-diffusion equation was used to determine the radial concentration of the solute species in a binary mixture undergoing a pressure gradient created by the ultracentrifugal force. ${ }^{33}$ This equation was also utilised to study transportation of the solute species in a binary mixture around an oscillating bubble excited by acoustic waves. ${ }^{34}$ Newman and Eirich ${ }^{35}$ modified the Langevin equation to describe the motion of a macromolecule in the solution exposed to an ultracentrifugal field, instead of Fick's equation, and determined the sedimentation constant. In another work, the reactiondiffusion dynamics was modeled by a spatiotemporal master equation constructed by a Markov state model for the diffusive part. $^{36}$

The effect of different types of acoustic fields on the spatial distribution of particles in a fluid has been extensively studied. ${ }^{37-40}$ The main objective of these studies is to calculate the radiation force applied on particles and mass transportation in acoustic fields but not their effect on the thermodynamics and kinetics of phase transition. The latter was considered mainly in the context of acoustic cavitation: (i) to model rectified diffusion of gas or vapour (evaporated at the liquid-bubble interface) towards an oscillating bubble leading to its gradual growth $^{41-44}$ and (ii) to model the segregation of a gas mixture inside an oscillating bubble driven by pressure and temperature gradients. ${ }^{44,45}$

Here we aim to study the theory of sonocrystallisation in a non-mass conserved system. We will develop and present a model which accounts for the effect of pressure and temperature oscillations on both aggregative and nonaggregative mechanisms. Furthermore, the developed model can describe the non-stationary kinetics of crystallisation in an arbitrary acoustic field, e.g., shock waves. The nucleation model developed in the preceding work is represented in Sec. II followed by the description of the cluster dynamics in Sec. III. We present equations for calculating mass transportation in an acoustic field in Sec. III B. A simplified form of this equation is then incorporated into both the Szilard and Fokker-Planck equations to achieve the kinetic model in a non-mass conserved system. Using this model, we determine the effect of different one-dimensional acoustic fields on the spatial distribution of monomers (Sec. V B). Subsequently, the nucleation work and kinetics are calculated and discussed (Sec. V C).

\section{CLUSTER MODEL AND THE WORK OF CLUSTER FORMATION}

The thermodynamics of phase transformation based on the Gibbs droplet model with an arbitrary dividing surface was studied in our preceding work. ${ }^{12}$ We quantified the effect of variation in pressure, temperature, and composition of the old phase on the work of formation of a cluster. We use the same model here to define clusters and to calculate the work of cluster formation in an acoustic field. The model is presented in this section without delving into derivations. The reader is referred to the previous work for the detailed discussion of the model.

Figure 1 schematically presents the cluster model. A phase transition takes place in the system which is considered to be a volume element coupled to a heat and particle bath. The choice of heat and particle bath essentially means that the temperature and volume of the system remain constant and the old phase in the system has the same chemical potential as that of the bath. Initially, the system consists of the homogeneous old phase. After cluster formation, the system includes three phases, namely, the core of the cluster taking the new phase (labeled with suffix $n$ ), the old phase surrounding the new phase, and an interface phase (labeled with suffix $\sigma$ ) which lies on an arbitrary dividing surface (DS) between the new and old phases. The interface phase is considered to be a zero volume layer, i.e., a Gibbs geometrical surface. For a given cluster size $n$, the properties of a cluster vary depending on the location of the DS. Here, we will use the new surface defined in the work of Haqshenas et al. ${ }^{12}$ as the DS. It is a non-equimolar dividing surface (non-EDS) identified by a dimensionless parameter $\lambda$ and the size-independent surface tension $\gamma_{\infty}$ which is the equilibrium macroscopic planar surface tension at the initial state. Assuming clusters are spherical, $\lambda=\delta / R_{0}$ is a dimensionless quantity that distinguishes an arbitrary dividing surface from the EDS, where $\delta$ is the radial separation between the EDS and the arbitrary DS ( $\delta=R^{E}-R$, where $R^{E}$ and $R$ are the radii of EDS and non-EDS clusters, respectively) and $R_{0}$ is the radius of a monomer in the new phase, considered to be a sphere. For an EDS, we have by definition $n_{\sigma}=0 .{ }^{13}$ As shown in the work of Haqshenas et al., ${ }^{12}$ the number of molecules in the interface phase for spherical clusters is obtained by

$$
n_{\sigma}=k_{\rho} \mathcal{G}\left(n_{n}\right)=k_{\rho} 3 \lambda\left(n_{n}^{2 / 3}+\lambda n_{n}^{1 / 3}+\frac{\lambda^{2}}{3}\right) .
$$

Here $k_{\rho}=1-\rho / \rho_{n}$ is a dimensionless quantity, where $\rho$ and $\rho_{n}$ are the molecular number density of the old phase and the new phase, respectively. The total size (in molecules) of a cluster then reads $n=n_{n}+n_{\sigma}=n_{n}+k_{\rho} \mathcal{G}\left(n_{n}\right)$. Depending on the density of new and old phases and the location of the dividing surface, $n_{\sigma}$ can be positive or negative.

Using this model to define a cluster of condensed phase, the work of cluster formation reads ${ }^{12}$

$$
\Delta \Omega=-n \Delta \mu+F_{\sigma, 1},
$$

where $F_{\sigma, 1}=n_{\sigma} \Delta \mu+\Omega_{\sigma}$ is the excess free energy, $\Omega$ is the thermodynamic grand potential, $\Delta \mu=\mu-\mu_{n}$ is the difference

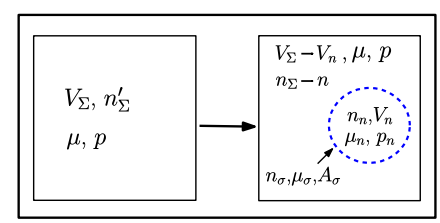

FIG. 1. Cluster formation in a system with constant volume, temperature, and chemical potential. $n_{\Sigma}^{\prime}$ and $n_{\Sigma}$ are the number of molecules in the system before and after cluster formation, respectively. Refer to the text for details. 
in chemical potentials of the old and new phases. $\Omega_{\sigma}$ is the grand potential associated with the interface phase which we represent by $A_{\sigma} \gamma_{\infty}$, where $A_{\sigma}$ is the interfacial surface area. We consider that the system is initially in the silent condition, i.e., there is no acoustic wave present, denoted by the nought suffix. At the initial temperature $T_{0}$ and pressure $p_{0}$, we can write $\Delta \mu=k_{B} T_{0} \ln \left(r_{0}\right)$, where $k_{B}$ is the Boltzmann constant and $r_{0}$ is the initial supersaturation ratio. For spherical clusters, the surface area becomes $A_{\sigma}=a_{0} \gamma_{\infty} n_{n}^{2 / 3}$, where $a_{0}$ is the surface area of a spherical monomer in the new phase. Substituting these formulas of $\Delta \mu$ and $A_{\sigma}$ in Eq. (2) yields the work of cluster formation at a reference silent condition as follows:

$$
\Delta \Omega_{0}=-n k_{B} T_{0} \ln \left(r_{0}\right)+n_{\sigma} k_{B} T_{0} \ln \left(r_{0}\right)+a_{0} \gamma_{\infty} n_{n}^{2 / 3}
$$

The cluster and nucleation models were validated by applying them to the test case of water droplet nucleation from vapour. $^{12}$ The work of the formation of the same size cluster when the pressure and temperature of the old phase fluctuate is given by ${ }^{12}$

$\Delta \Omega\left(n_{n}, n_{\sigma}, p, T\right)=\Delta \Omega_{0}\left(n_{n}, n_{\sigma}\right)-\int_{T_{0}}^{T} \Delta s_{e x c} d T-\int_{p_{0}}^{p} v \Delta n_{e x c} d p$,

where $\Delta \Omega_{0}\left(n_{n}, n_{\sigma}\right)$ is given in Eq. (3), $\Delta s_{\text {exc }}=-s\left(n_{n}+n_{\sigma}\right)$ $+n_{n} s_{n}+n_{\sigma} s_{\sigma}$ is the excess entropy gained by the system through the formation of a cluster of size $n$ ( $s$ represents entropy per number of molecules and the suffices are explained above), and $\Delta n_{\text {exc }}=k_{\rho} n_{n}+n_{\sigma}$ is the excess number of molecules in the cluster. When the new phase is denser than the old phase, we have $k_{\rho}>0$ and consequently $\Delta n_{\text {exc }}>0$ and vice versa. In the case of an incompressible solution and isothermal condition, this equation simplifies to

$$
\Delta \Omega\left(n_{n}, n_{\sigma}, p\right)=\Delta \Omega_{0}\left(n_{n}, n_{\sigma}\right)-\Delta n_{e x c} v \Delta p
$$

where $v=1 / \rho$ is the specific volume of a molecule in the old phase and $\Delta p=p-p_{0}$ is the variation in pressure compared to the reference pressure $p_{0}$. The clustering work under this circumstance is obtained by substituting Eq. (3) into (5) which reads

$$
\begin{aligned}
\Delta \Omega\left(n_{n}, n_{\sigma}, p\right)= & -n k_{B} T_{0} \ln \left(r_{0}\right)+n_{\sigma} k_{B} T_{0} \ln \left(r_{0}\right) \\
& -\Delta n_{\text {exc }} v \Delta p+a_{0} \gamma_{\infty} n_{n}^{2 / 3} .
\end{aligned}
$$

These equations will be used to determine the kinetics of cluster formation in Sec. III.

\section{KINETICS OF NUCLEATION}

The Szilard model explains cluster formation as a result of a series of consecutive attachments and detachments of monomers. This model describes the kinetics of nucleation, the early stage of growth, and even the Ostwald-ripening regime ${ }^{46}$ as they are mainly driven by gaining and losing monomers. The Szilard model is expressed by
For $n=1$ :

$$
\begin{aligned}
\frac{d Z_{1}(t)}{d t}= & -2 f_{1}(t) Z_{1}(t)+2 g_{2}(t) Z_{2}(t)+\sum_{n=3}^{N} g_{n}(t) Z_{n}(t) \\
& -\sum_{n=2}^{N} f_{n}(t) Z_{n}(t)+K_{1}(t)-L_{1}(t)
\end{aligned}
$$

for $n \geq 2$ :

$$
\begin{aligned}
\frac{d Z_{n}(t)}{d t}= & f_{n-1}(t) Z_{n-1}(t)-g_{n}(t) Z_{n}(t)-f_{n}(t) Z_{n}(t) \\
& +g_{n+1}(t) Z_{n+1}(t)+K_{n}(t)-L_{n}(t),
\end{aligned}
$$

where $f_{n}(t)$ and $g_{n}(t)$ are attachment and detachment frequencies at time $t, Z_{n}(t)$ is the concentration of $n$-sized clusters, and $K_{n}(t)-L_{n}(t)$ reflects the non-aggregative change in the concentration of the cluster size $n$ in a non-mass conserved system. $K_{n}(t)$ is the inward flux of $n$-sized clusters to the system from the bath and $L_{n}(t)$ is the outward flux of $n$-sized clusters to the bath from the system. The Szilard model is a discrete equation. The truncated second order Taylor expansion of this discrete equation about point $n$ produces the continuous form of the Szilard model which is known as the Fokker-Planck Equation (FPE) and reads

$$
\begin{aligned}
\frac{\partial Z(n, t)}{\partial t}= & -\frac{\partial}{\partial n}\left(v(n, t) Z(n, t)-\frac{1}{2} \frac{\partial[d(n, t) Z(n, t)]}{\partial n}\right) \\
& +K(n, t)-L(n, t),
\end{aligned}
$$

where $v(n, t)$ and $d(n, t)$ are given by

$$
\begin{aligned}
& v(n, t)=f(n, t)-g(n, t), \\
& d(n, t)=f(n, t)+g(n, t) .
\end{aligned}
$$

$v(n, t)$ is the drift velocity along the size axis, known as the mean growth rate, specifying the rate of deterministic incrementation of the cluster size $n . d(n, t)$ is the rate of the random change of cluster size along the size axis (dispersion of cluster size along the size axis). The FPE is computationally favoured if the concentration of large clusters is desired. However, because of approximation in the derivation of FPE, it is inaccurate with respect to the Szilard equation at small clusters. Therefore a hybrid model is envisaged to take advantages of both discrete and continuous descriptions of the cluster dynamics. ${ }^{47}$ Subsequently, the cluster size axis $n$ is divided up to two sections, a discrete part $n=1, \ldots, N_{d}$ and a continuous part $n=\left[N_{d}+1, N\right]$, where $N_{d}$ is the boundary between discrete and continuous sections and $N$ is the largest cluster size postulated. $N_{d}$ is chosen such that the simulation results are independent of this choice and the FPE numerically converges to the result of Szilard model. A boundary condition of the continuity of cluster flux is applied at the transition point between the discrete and continuous models. The cluster flux along the size axis is defined as

$$
j_{n}^{c}(t)=f_{n}(t) Z_{n}(t)-g_{n+1}(t) Z_{n+1}(t) .
$$

The hybrid method is schematically illustrated in Fig. 2.

In our previous study, we assumed that the system conserves mass. As such, for both discrete and continuous models, we set $K(n, t)=L(n, t)=0$. In this work, we relax this assumption and include the effect of mass transportation mediated by wave propagation. We will determine the net flux of clusters to 


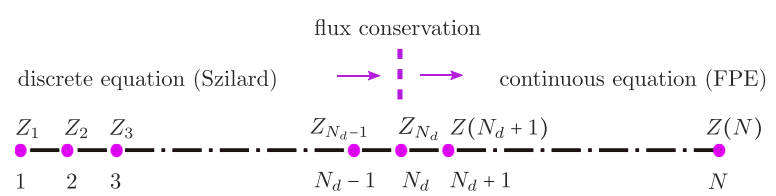

FIG. 2. Schematic representation of the hybrid model. $N_{d}$ is the boundary between discrete and continuous sections and $N$ is the largest cluster size postulated. $Z_{n}(t)$ is the concentration of $n$-sized clusters.

the system, i.e., the term $K_{n}-L_{n}$ in Eqs. (7) and (8), resulting from wave propagation. To this end, the solution is modeled as a mixture of the solute and solvent species. Therefore, in Sec. III A, we will study the continuity equation for a mixture and then proceed with the calculation of forced diffusion created by wave propagation.

\section{A. Conservation of mass in a mixture}

Here, we consider only the contribution of mass transportation to the kinetics of cluster formation, i.e., only the non-aggregative mechanism in the kinetics of population evolution without accounting for the coalescence of clusters. The system, see Fig. 1, is a volume element fixed in space, and its position relative to the origin of a fixed system of coordinates is specified by the position vector $\mathbf{r}$. All the physical variables such as density, pressure, and temperature will therefore be functions of space and time. Denoting the properties of the solution, the solvent and the solute with suffices $m, 1$, and 2 , respectively, the mass density of the solution reads $\rho_{m}(t, \mathbf{r})$ $=\rho_{1}(t, \mathbf{r})+\rho_{2}(t, \mathbf{r})$. The conservation of mass for the solution is given by ${ }^{31}$

$$
\frac{\partial \rho_{m}(t, \mathbf{r})}{\partial t}=-\nabla \cdot\left(\rho_{m}(t, \mathbf{r}) \mathbf{u}\right)
$$

which in the case of an incompressible fluid, i.e., a solution with constant mass density, becomes

$$
\nabla \cdot \mathbf{u}=0
$$

Here $\mathbf{u}$ is the velocity vector in the Cartesian coordinate system. Considering the cluster size as a discrete variable, we can write the mass density of the solute species in terms of concentration of different clusters as follows:

$$
\rho_{2}(t, \mathbf{r})=\frac{M_{2}}{\mathcal{N}_{\mathcal{A}}} \sum_{n=1}^{N(t)} n Z_{n}(t, \mathbf{r})=\sum_{n=1}^{N(t)} Y_{n}(t, \mathbf{r}),
$$

where $Y_{n}(t, \mathbf{r})=\frac{M_{2}}{\mathcal{N}_{\mathcal{A}}} n Z_{n}(t, \mathbf{r})$ is the mass density of $n$-sized clusters in the solution, $M_{2}$ is the molar mass of the solute, and $\mathcal{N}_{\mathcal{A}}$ is Avogadro's number. This equation states that the solute can be considered to be made of $N(t)$ species with mass densities of $Y_{n}(t), n=1, \ldots, N(t)$. The quantity $Z_{n}$ (or $Y_{n}$ ), and as a result $\rho_{2}$, changes in the system because of mass transportation. Hereafter we will use the superscript $m t$ to distinguish the effect of mass transportation. Also, the notation $(t, \mathbf{r})$ will be omitted for the sake of brevity, though concentrations and thermodynamic state variables vary temporally and spatially. The conservation of mass for the $n$th component in the solution, where $n=1, \ldots, N(t)$, is given by ${ }^{31}$

$$
\frac{\partial}{\partial t} Y_{n}^{m t}=-Y_{n}^{m t}(\nabla \cdot \mathbf{u})-\mathbf{u} \cdot \nabla Y_{n}^{m t}-\nabla \cdot \mathbf{j}_{[\mathbf{n}]} .
$$

Here $\mathbf{j}_{[n]}$ is the molecular mass flux of clusters of size $n$ through space and can be obtained using the generalized Fick's equation in a multi-component system. Invoking the definition of $Y_{n}$ and dividing both sides by $n M_{2} / \mathcal{N}_{\mathcal{A}}$, we arrive at the concentration form of this equation as follows:

$$
\frac{\partial}{\partial t} Z_{n}^{m t}=-Z_{n}^{m t} \nabla \cdot \mathbf{u}-\mathbf{u} \cdot \nabla Z_{n}^{m t}-\frac{\mathcal{N}_{\mathcal{A}}}{M_{2} n} \nabla \cdot \mathbf{j}_{[\mathbf{n}]} .
$$

This equation expresses the rate of change in $Z_{n}$ as a result of different mass transport mechanisms: the convective flux (the first two terms on the RHS) and the molecular flux of the solute species (the last term on the RHS). Convection represents mass transportation due to the average velocity of all molecules which is the average velocity of the fluid as a whole. Diffusion is viewed as mass transportation due to the instantaneously changing stochastic velocity of individual molecules, compared to the averaged fluid velocity. For the transportation of dilute species in a mixture where the solvent dominates the momentum of the system, we can consider a reference velocity equal to the velocity of the dominant component, i.e., the solvent here, and identify a diffusive flux and a convective flux accordingly.

The change in the concentration of clusters due to nonaggregative mechanism is therefore given by

$$
K_{n}(t, \mathbf{r})-L_{n}(t, \mathbf{r})=\frac{\partial}{\partial t} Z_{n}^{m t}(t, \mathbf{r}) .
$$

Replacing the RHS by Eq. (16) gives the discrete representation of the net mass flux in the system. The equations derived in this section are in a generic format and account for the variation in the concentration of clusters of different sizes because of mass convection and diffusion. A simpler form of this equation can be obtained if we impose some assumptions. Such an approximation is discussed in Sec. III A 1.

\section{Simplified conservation of mass equation}

Considering that the concentration of monomers in the system at the initial time and during nucleation is significantly greater than that of $n$-mers, we can postulate that the solute species is mainly constituted of monomers. This approximates the solution as a binary mixture of the single species solute of monomers and the solvent. This approximation is also supported noting that larger clusters are less mobile as the diffusivity is inversely proportional to $n^{1 / 3}$. Moreover, it was shown in Ref. 48 in a closed system, the presence of $n$-sized clusters, $2 \leq n \leq 4$, would change the nucleation rate by less than one order of magnitude. Thus, the monomer mass flux $\mathbf{j}_{[1]}$ is required to determine mass transportation which reads (see Appendix A)

$$
\begin{aligned}
\mathbf{j}_{[1]}= & -\frac{M_{1} M_{2} D}{M_{m} \mathcal{N}_{\mathcal{A}}} \nabla Z_{1}^{m t}-D_{2}^{T} \nabla \ln T \\
& -\frac{M_{1} M_{2}^{2} D}{M_{m} \mathcal{N}_{\mathcal{A}} \mathcal{R} T} Z_{1}^{m t}\left(\frac{\overline{V_{2}}}{M_{2}}-\frac{1}{\rho_{m}}\right) \nabla p,
\end{aligned}
$$


where $D$ and $D_{2}^{T}$ are the mass and thermal diffusivity of monomers, respectively, $\overline{V_{2}}=M_{2} / \rho_{2}$ is the partial molal volume, $\mathcal{R}$ is the gas constant, and $M_{m}$ and $M_{1}$ are the molar mass of the solution and solvent, respectively. In the absence of external forces, the mass flux of monomers consists of three contributions, ${ }^{31}$ namely, ordinary diffusion driven by the spatial concentration gradient of species (the first term on the RHS), thermal diffusion term (the second term on the RHS) which expresses mass diffusion because of the temperature gradient, and pressure diffusion (the third term on the RHS) which describes the tendency for the mass flux under the influence of pressure gradient. The ratio of thermal diffusivity over (mass) diffusivity is called the Soret coefficient, i.e., $S^{T}=D_{2}^{T} / D$.

The effect of a temperature gradient on mass transport is usually considered negligible unless there is a steep temperature gradient. ${ }^{31}$ Thus, this term can be neglected as far as acoustic wave propagation in the solution is concerned. In the case of inertial cavitation, a substantial temperature rise at the centre of the collapsing bubble occurs which vanishes rapidly across space from the centre of the bubble as time progresses. ${ }^{11,44,49}$ However, the temperature rise in the solution in the vicinity of the surface of the bubble is much lower. It is close to the temperature of the solution rather than that of the core of the bursting bubble. ${ }^{50}$ In contrast, the pressure gradient in the vicinity of the bursting bubble is substantially greater than the temperature gradient. ${ }^{44,49-52}$ Furthermore, for an organic mixture and an aqueous solution, the absolute value of the Soret coefficient is of the order of $\left|S^{T}\right| \approx 0.001-0.01 \mathrm{~K}^{-1} .{ }^{53}$ Consequently, the contribution of the thermal diffusion term to mass transportation tends to be much smaller than that of the concentration and pressure terms and can therefore be overlooked. Equation (18) then reduces to

$$
\mathbf{j}_{[1]}=-\frac{M_{2} D}{\mathcal{N}_{\mathcal{A}}}\left(\frac{M_{1}}{M_{m}} \nabla Z_{1}^{m t}+\frac{M_{1}}{M_{m}} \frac{k_{j}^{p}}{T} Z_{1}^{m t} \nabla p\right),
$$

where $k_{j}^{p}=\frac{M_{2}}{\mathcal{R}}\left(\frac{\overline{V_{2}}}{M_{2}}-\frac{1}{\rho_{m}}\right)$. In general, the densities and consequently $k_{j}^{p}$ are time and space dependent, which makes the above equation nonlinear.

Considering that the solution is dilute, we can write $M_{m}$ $\approx M_{1}$ and $\rho_{m} \approx \rho_{1}$; hence, the above equation becomes

$$
\mathbf{j}_{[1]}=-\frac{M_{2} D}{\mathcal{N}_{\mathcal{A}}}\left(\nabla Z_{1}^{m t}+\frac{k_{j}^{p}}{T} Z_{1}^{m t} \nabla p\right) .
$$

Equations developed thus far in this section are based on the assumption that the solute species is present mainly in the form of monomers with a population considerably greater than $n$-mers. This literally transforms mass transportation into monomer transportation which yields

$$
\begin{aligned}
& K_{1}-L_{1}=\frac{\partial}{\partial t} Z_{1}^{m t}, \\
& K_{n}-L_{n}=0, \quad n \geq 2 .
\end{aligned}
$$

Likewise, in the theory of thin film condensation, the system is usually assumed non-mass conserved by including the arrival and evaporation of monomers in the kinetics model of the cluster formation. ${ }^{14-19}$

The net rate of monomer transportation in an incompressible solution is obtained by combining Eqs. (13), (20), and (16) which reads

$$
\frac{\partial}{\partial t} Z_{1}^{m t}=-\mathbf{u} \cdot \nabla Z_{1}^{m t}+D \nabla \cdot\left(\nabla Z_{1}^{m t}+\frac{k_{j}^{p}}{T} Z_{1}^{m t} \nabla p\right) .
$$

The assumption of incompressibility for wave propagation in an aqueous solution holds true for a vast range of excitation pressures and frequencies. ${ }^{8}$ However, this condition may fail when the pressure perturbation is generated from a violent inertial cavitation or at a strong shock front with associated acoustic Mach number of unity or higher. ${ }^{8,54}$ Equation (22) is highly nonlinear. In favour of simplification in the mathematical implementation, following the work of Louisnard et al. ${ }^{34}$ in the limit of a dilute and incompressible solution, we can consider making an additional approximation where $k_{j}^{p}$ is constant and only depends on the solution properties.

\section{B. Hybrid model with mass transportation}

Equations (16) and (17) give the variation in the concentration of $n$-sized clusters because of mass transportation in a non-mass conserved system. Considering the discrete representations of cluster size, substituting these relationships into Eq. (7) yields the Szilard equation which determines the kinetics of cluster formation in a non-mass conserved system. This equation for an $n$-sized cluster reads

$$
\begin{aligned}
\frac{\partial}{\partial t} Z_{n}= & f_{n-1}(t) Z_{n-1}-g_{n}(t) Z_{n}-f_{n}(t) Z_{n}+g_{n+1}(t) Z_{n+1} \\
& -Z_{n} \nabla \cdot \mathbf{u}-\mathbf{u} \cdot \nabla Z_{n}-\frac{\mathcal{N}_{\mathcal{A}}}{M_{2} n} \nabla \cdot \mathbf{j}_{[\mathbf{n}]} .
\end{aligned}
$$

The terms on the RHS are divided into two main categories: the first four terms on the RHS which represent the aggregative change in the cluster concentration and the last three terms on the RHS indicating the non-aggregative change in the cluster concentration governed by the convective and diffusive transportation of clusters. The continuous format of this equation (the FPE with mass transportation) is derived in Appendix B and reads

$$
\begin{aligned}
\frac{\partial}{\partial t} Z(n)= & -\frac{\partial}{\partial n}\left(v(n) Z(n)-\frac{1}{2} \frac{\partial[d(n, t) Z(n)]}{\partial n}\right) \\
& -Z(n) \nabla \cdot \mathbf{u}-\mathbf{u} \cdot \nabla Z(n)-\frac{\mathcal{N}_{\mathcal{A}}}{M_{2} n}\left(\nabla \cdot \mathbf{j}_{[\mathrm{n}]}\right) .
\end{aligned}
$$

For the simplified case presented in Sec. III A 1 where the solute species is mainly made of monomers, we can construct the following hybrid model:

$$
\begin{aligned}
& \text { for } n=1: \\
& \begin{aligned}
\frac{\partial}{\partial t} Z_{1}= & -2 f_{1}(t) Z_{1}+2 g_{2}(t) Z_{2}+\sum_{n=3}^{N} g_{n}(t) Z_{n}-\sum_{n=2}^{N} f_{n}(t) Z_{n} \\
& -Z_{1} \nabla \cdot \mathbf{u}-\mathbf{u} \cdot \nabla Z_{1}-\frac{\mathcal{N}_{\mathcal{A}}}{M_{2}} \nabla \cdot \mathbf{j}_{[1]},
\end{aligned}
\end{aligned}
$$


for $1<n \leq N_{d}$ :

$$
\frac{\partial}{\partial t} Z_{n}=f_{n-1}(t) Z_{n-1}-g_{n}(t) Z_{n}-f_{n}(t) Z_{n}+g_{n+1}(t) Z_{n+1},
$$

for $N_{d}<n \leq N$ :

$$
\frac{\partial}{\partial t} Z(n)=-\frac{\partial}{\partial n}\left(v(n, t) Z(n)-\frac{1}{2} \frac{\partial[d(n, t) Z(n)]}{\partial n}\right),
$$

where the monomer mass flux $\mathbf{j}_{[1]}$ is given in Eq. (18).

From the perspective of thermodynamics and fluid mechanics, the following assumptions are made in this hybrid model: (i) cluster formation is governed by the association and depletion of monomers, (ii) monomers have the same convective velocity as that of the flow and the no-slip condition holds, and (iii) the solution is a continuum medium modeled as a binary mixture. Since none of these assumptions restrict the temporal application of these equations, this hybrid model should be valid for describing the cluster dynamics during transient processes, e.g., under a rapid pressure fluctuation in the vicinity of a collapsing bubble or shock waves.

This model tells that the concentration change over time is determined by two fluxes, the flux along the size axis $n$ representing the aggregative process, see Eq. (11), and the mass flux along the space axis $\mathbf{r}$ modeling the contribution of the non-aggregative mechanism, Eq. (17). In a massconserved system, the latter flux disappears and the flux of clusters over the size axis (aggregative mechanism) will be the only driving mechanism of the cluster dynamics. It should be noted that these two mechanisms are coupled because intercluster transition frequencies are functions of the concentration of monomers, which will be shown in Sec. IV. This implies that these fluxes cannot be solved for independently. Nonetheless, the question that arises here is whether or not these two fluxes occur at comparable rates? If not, it would be physically reasonable to overlook the slower process in favour of further simplifications. This is further discussed in Sec. V C.

In the special case of an incompressible solution, the first line of this hybrid model, i.e., Eq. (25a), can be further simplified using Eq. (22) which becomes

$$
\begin{aligned}
\frac{\partial}{\partial t} Z_{1}= & -f_{1}(t) Z_{1}+2 g_{2}(t) Z_{2}+\sum_{n=3}^{N} g_{n}(t) Z_{n}-\sum_{n=2}^{N} f_{n}(t) Z_{n} \\
& -\mathbf{u} \cdot \nabla Z_{1}+D \nabla \cdot\left(\nabla Z_{1}+\frac{k_{j}^{p}}{T} Z_{1} \nabla p\right) .
\end{aligned}
$$

Finally, neglecting the Ostwald ripening process, this equation can be further simplified to

$$
\begin{aligned}
\frac{\partial}{\partial t} Z_{1}= & -f_{1}(t) Z_{1}+g_{2}(t) Z_{2}-\mathbf{u} \cdot \nabla Z_{1} \\
& +D \nabla \cdot\left(\nabla Z_{1}+\frac{k_{j}^{p}}{T} Z_{1} \nabla p\right) .
\end{aligned}
$$

In summary, Eqs. (25a), (26), and (27) allow the determination of the concentration of monomers in a non-mass conserved system for the following situations, respectively: (i) accounting for the Ostwald ripening, the compressibility of the solution and all mechanisms contributing to mass transportation, (ii) accounting for the Ostwald ripening but not the Soret effect and the compressibility of the solution which is assumed to be dilute, and (iii) overlooking the Ostwald ripening process, the Soret effect, and the compressibility of the dilute solution. Each of these situations can be taken to study the kinetics of the cluster formation in a non-mass conserved system under sonication when the underlying assumptions hold. For instance, the Ostwald ripening takes place towards the end of nucleation when the supersaturation ratio is reduced significantly and the concentration of large clusters is considerable. Therefore, Eq. (27) should adequately model the effect of lower amplitude excitations, or weak transient pressure waves, on the kinetics of nucleation (see the work of Haqshenas et al. ${ }^{12}$ for more information about the Ostwald ripening in an acoustic field). To solve the hybrid model, we need to determine the cluster transition frequencies which are addressed in Sec. IV.

\section{CLUSTER TRANSITION FREQUENCIES}

The attachment of monomers to a condensed-phase cluster depends on the state of the old phase. The governing mechanisms of monomer attachment in solutions are as follows: ${ }^{13}$ (i) the volume or surface diffusion of molecules and (ii) the interface transfer. Both mechanisms may take place depending on the cluster size. Studying the homogeneous nucleation of solids from a dilute solution exposed to acoustic waves, we assume that the volume diffusion is the dominant monomer attachment mechanism for all cluster sizes. Here, a continuum model of the volume diffusion is taken which is formulated as follows for a spherical $n$-sized condensed cluster: ${ }^{13}$

$$
f_{n}(t)=k_{f}\left(n_{n}\right) Z_{1}(t)
$$

where

$$
k_{f}\left(n_{n}\right)=4 \pi \alpha_{n} D R_{0}\left(1+n_{n}^{-1 / 3}\right)\left(1+n_{n}^{1 / 3}\right),
$$

where $\alpha_{n}$ is the sticking coefficient which is nearly unity in a dilute solution and $R_{0}$ is the radius of a monomer in the new phase, considered to be a sphere. Here, both the cluster and monomers are considered to be mobile and to diffuse through the medium. The Stokes-Einstein equation is used to calculate the diffusivity of a cluster. The diffusivity and concentration of monomers are the main factors affecting the attachment rate. The effect of pressure on diffusivity is almost negligible due to weak pressure dependence of viscosity and incompressibility of solution. Mass transportation influences the spatial concentration of monomers; hence, the attachment frequency varies temporally and spatially.

The rate at which monomers detach from an $n$-sized cluster can be estimated following the Zeldovich method which is based on the detailed balance at the equilibrium state, i.e., $j_{n, e q}^{c}=0$. Employing the Zeldovich method and considering that the cluster size is a discrete variable, the detachment frequency for the case of time-varying chemical potential of the old phase and a quasi-equilibrium condition reads ${ }^{55}$

$$
g_{n}(t)=f_{n-1}(t) \exp \left(\frac{\Delta \Omega_{n}(t)-\Delta \Omega_{n-1}(t)}{k_{B} T(t)}\right) .
$$


Substituting Eqs. (3), (5), and (28) into this equation gives a generic equation for the detachment frequency in an acoustic wave field; see the work of Haqshenas et al. ${ }^{12}$ The simplified form of this equation for a cluster defined by the new surface in a dilute and incompressible solution exposed to isothermal pressure fluctuations reads

$$
\begin{aligned}
g_{n}(p, T, x)= & k_{f}\left(n_{n}\right) C_{e} r_{0}^{n_{\sigma}^{\prime}\left(n_{n}\right)} \exp \left(\frac{2}{3} \frac{a_{0} \gamma_{\infty}}{k_{B} T_{0}} n_{n}^{-1 / 3} n_{n}^{\prime}\left(n_{n}\right)\right) \\
& \times \exp \left(-\frac{v \Delta p}{k_{B} T_{0}} \Delta n_{\text {exc }}^{\prime}\right),
\end{aligned}
$$

where $C_{e}$ is the equilibrium concentration at temperature $T_{0}$ (solubility). The approximation of $r_{0} \approx Z_{1} / C_{e}$ was used in this equation which is justified as the solute is mainly comprised of monomers at the initial stage. $\Delta n_{e x c}^{\prime}=1-\frac{\rho}{\rho_{n}} n_{n}^{\prime}\left(n_{n}\right)$ and quantities $n_{\sigma}^{\prime}\left(n_{n}\right)$ and $n_{n}^{\prime}\left(n_{n}\right)$ are the first derivatives of $n_{\sigma}$ and $n_{n}$ with respect to the cluster size $n$, respectively, which are obtained using Eq. (1). As shown in the work of Haqshenas et al.,$^{12}$ these equations are valid for both discrete and continuous cluster size variables and hence can be used for all equations in the hybrid model.

\section{SIMULATION RESULTS}

We have established the required equations to determine the kinetics of nucleation while accounting for the effect of fluctuations in the thermodynamic state of the old phase. Our objective in this section is to evaluate our new model in a nonmass conserved system exposed to acoustic waves. Simulations in a mass-conserved system were already presented in the work of Haqshenas et al., ${ }^{12}$ and the numerical implementation of the hybrid model was validated there.

In all the simulations presented here, the reference state is considered to be at room temperature and atmospheric pressure, $T_{0}=293 \mathrm{~K}$ and $p_{0}=0.1 \mathrm{MPa}$, respectively, and sonocrystallisation in an aqueous environment is considered to be an isothermal process. The physicochemical properties of the solution, the new phase, and water at the reference state are listed in Tables II and III in Appendix C. These properties are the same as those used in our previous work. ${ }^{12}$ Likewise, the initial supersaturation is $r_{0}=30$, and clusters are defined to be non-EDS with $\lambda=0.35$, unless otherwise stated.

In a non-mass conserved system, the fluid dynamics must be calculated to determine mass transportation in the system. The system's characteristics (e.g., boundary conditions) influence the fluid dynamics in the system. Therefore, a similar acoustic source tends to produce different effects on a phase transition in different systems. We will consider various scenarios of one-dimensional wave propagation in the bath. Considering one-dimensional wave propagation in an unbounded system, the simulations presented here does not account for acoustic streaming. This section is continued by calculating acoustic wave propagation in the solution followed by the determination of mass transportation. Subsequently, the kinetics of crystallisation in a non-mass conserved system are computed and discussed.

\section{A. Acoustic wave propagation}

The equations derived in this work are not limited to a specific source type and are generic in this sense. For the sake of simplicity, we consider finite amplitude plane acoustic waves emitted from an infinite plate oscillating harmonically in the direction normal to the plate. The radiated waves propagate through a semi-infinite dissipative and nonlinear aqueous medium. We will consider up to the second order approximation in the equation of state for density terms. Due to the nonlinearity of the medium, the wave can distort and form a shock wave. The simplest second order wave equation which describes the combined effects of dissipation and nonlinearity on the propagation of plane waves is Burgers' equation. ${ }^{8}$ Burgers' equation can be formulated in the following format: ${ }^{.6}$

$$
\begin{aligned}
& \frac{\partial v}{\partial t}+v \frac{\partial v}{\partial \xi}=\frac{\delta_{0}}{2} \frac{\partial^{2} v}{\partial \xi^{2}}, \\
& v=u+c-c_{0}, \\
& \xi=x-c_{0} t, \\
& \delta_{0}=\frac{\eta}{\rho_{1}}\left(\frac{4}{3}+\frac{\eta_{B}}{\eta}+\frac{\left(\gamma_{s}-1\right) \kappa}{\eta C_{p}}\right),
\end{aligned}
$$

where $u$ is the fluid particle velocity, $c$ is the local wave speed, $c_{0}$ is the wave speed in the initial undisturbed condition, $x$ is the distance from the source, $\xi$ is the retarded distance (spatial propagation delay), $\delta_{0}$ is the sound diffusivity, $\eta$ is the dynamic (shear) viscosity, $\eta_{B}$ is the bulk viscosity, $\gamma_{s}$ is the specific heat ratio, $\kappa$ is the thermal conductivity, and $C_{p}$ is the specific heat at constant pressure. With respect to the dilute solution assumption, we postulate that the hydrodynamic properties of the wave medium are similar to those of the solvent. This is a legitimate assumption within the range of the driving frequencies which will be used in this work, i.e., $f \leq 2 \mathrm{MHz}$. The source oscillates harmonically with an angular frequency of $\omega=2 \pi f$ and a velocity magnitude of $u_{m}$. This results in the boundary condition of $p(t, 0)=p_{m} \sin (\omega t)$, where $p_{m} / u_{m}=\rho_{1} c_{0}$. The latter uses the specific acoustic impedance relationship for plane waves.

Burgers' equation is widely used to calculate the progressive plane wave field in a lossy and nonlinear medium within the limitation of the weak shock theory. The condition for the weak shock is met if $M_{a} \ll 1$, where $M_{a}$ $=u_{0} / c_{0}$ is the acoustic Mach number. We will solve Burgers' equation and check this condition for the range of parameters used in this work. Several methods have been developed to solve Burgers' equation analytically and numerically in either the frequency domain or the time domain. Here, we will use Mitome's exact solution ${ }^{56}$ which allows determination of pressure in the pre- and post-shock regions in the time domain. The derivation and the numerical implementation of Mitome's solution are skipped here for the sake of brevity, and the reader is referred to Ref. 57 for more details.

A relatively strong pressure field is required to considerably influence the thermodynamics of phase transition. ${ }^{12}$ Considering the simulations presented in the preceding work, ${ }^{12}$ we set $p_{m}=50 \mathrm{MPa}$ and calculate the pressure field. 


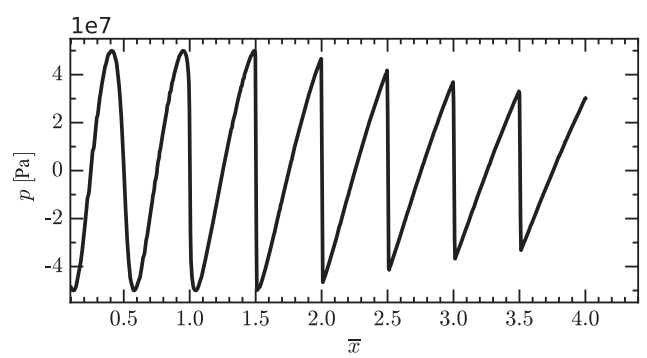

FIG. 3. Plane wave field across space up to $4 x_{s}$ at $\tau_{w}=2 \pi$ with $f=1 \mathrm{MHz}$ and $p_{m}=50 \mathrm{MPa}$.

Figure 3 shows the wave propagating across space at the end of an excitation period for the driving condition $f=1 \mathrm{MHz}, p_{m}$ $=50 \mathrm{MPa}$. The abscissa is the non-dimensionalised distance with respect to the lossless shock distance, i.e., $\bar{x}=x / x_{s}$, where $x_{s}=c_{0} / \beta \omega M_{a}=c_{0}^{2} / \beta \omega u_{m}$ and $\beta$ is the coefficient of nonlinearity. We can see in the pre-shock region, i.e., $\bar{x}<1$, the wave field can be safely approximated by a harmonic traveling wave as the distortion is negligible. However, around the shock formation distance (henceforth, shock distance), the waveform distorts and gradually develops into an $N$-shaped waveform (henceforth, $N$-wave) in the far field. Thus, as long as $x \ll x_{s}$, the harmonic traveling wave equation is sufficient to accurately represent the acoustic field. The acoustic Mach number for the wave shown in Fig. 3 is $M_{a}=0.02$ which satisfies the weak shock condition.

The temperature in an aqueous medium while only accounting for the absorption of a plane wave energy was determined before, ${ }^{8,57}$ confirming that the plane wave propagation can be considered to be isothermal if (i) the system is located in the pre-shock zone or (ii) the acoustic irradiation is short or pulsed for systems in $x>x_{s}$. Simulation parameters will be chosen such that the isothermal condition holds for the results presented in this work.

\section{B. Mass transportation in the acoustic wave field}

We will study mass transportation created by acoustic waves in the system, without a phase transformation, for different excitations by solving the non-dimensionalised form of Eq. (22). We will then solve the coupled problem of sonocrystallisation in a non-mass conserved system for the situation where mass transportation is more considerable.

The simplified non-dimensionalised equation of mass transportation in a one-dimensional acoustic field reads (the derivation is presented in supplementary material)

$$
\frac{\partial}{\partial \tau} \overline{Z_{1}}=-\bar{u} \frac{\partial \overline{Z_{1}}}{\partial \overline{x_{d}}}+\frac{1}{P e} \frac{\partial^{2} \overline{Z_{1}}}{\partial{\overline{x_{d}}}^{2}}+\frac{\bar{\beta}}{P e} \frac{\partial \overline{Z_{1}}}{\partial \overline{x_{d}}} \frac{\partial \bar{p}}{\partial{\overline{x_{d}}}^{2}}+\frac{\bar{\beta}}{P e} \overline{Z_{1}} \frac{\partial^{2} \bar{p}}{\partial{\overline{x_{d}}}^{2}} .
$$

Here $\overline{x_{d}}$ refers to the dimensionless location.

We will initially present the simulation results of the transportation of monomers in an acoustic field with different pressure amplitudes and frequencies. We will then proceed to determine the kinetics of crystallisation over time and across space for a case where mass transportation is more substantial. The acoustic pressure amplitude is chosen to be $10 \mathrm{MPa}$ and
TABLE I. Non-dimensionalisation constants. The details are explained in the supplementary material.

\begin{tabular}{lcccccc}
\hline \hline & $\begin{array}{c}t_{c} \\
(\mathrm{~ns})\end{array}$ & $\begin{array}{c}l_{c} \\
(\mathrm{~nm})\end{array}$ & $\begin{array}{c}v_{c} \\
\left(\mathrm{~m} \mathrm{~s}^{-1}\right)\end{array}$ & $\begin{array}{c}p_{c} \\
(\mathrm{MPa})\end{array}$ & $\begin{array}{c}P e \\
(\mathrm{DL})\end{array}$ & $\begin{array}{c}\bar{\beta} \\
(\mathrm{DL})\end{array}$ \\
\hline $\begin{array}{l}p_{m}=10 \mathrm{MPa}, \\
f=1 \mathrm{MHz}\end{array}$ & 6.6 & 44.7 & 6.75 & 10 & 302 & -0.12 \\
\hline $\begin{array}{l}p_{m}=50 \mathrm{MPa}, \\
f=1 \mathrm{MHz}\end{array}$ & 1.32 & 44.7 & 33.74 & 50 & 1509 & -0.61 \\
\hline $\begin{array}{l}p_{m}=50 \mathrm{MPa}, \\
f=100 \mathrm{kHz}\end{array}$ & 4.18 & 141.4 & 33.74 & 50 & 4773 & -0.61 \\
\hline \hline
\end{tabular}

$50 \mathrm{MPa}$. Simulations with $p_{m}=50 \mathrm{MPa}$ were conducted in two different frequencies of $100 \mathrm{kHz}$ and $1 \mathrm{MHz}$. This range of acoustic parameters is selected in harmony with parameters used in our previous work. ${ }^{12}$

The values of non-dimensionalisation constants for different excitation parameters are shown in Table I. The density of the solute species in an aqueous solution typically falls in the range of $1000<\rho_{\text {mass }, 2}<2500 \mathrm{~kg} \mathrm{~m}^{-3}$. We choose $\rho_{\text {mass }, 2}=2000 \mathrm{~kg} \mathrm{~m}^{-3}$ and employ the following equation to estimate the molar mass for the solute species, i.e., $M_{2}=4 \pi R_{0}^{3} \mathcal{N}_{\mathcal{A}} \rho_{\text {mass }, 2} / 3=\mathcal{N}_{\mathcal{A}} v_{n} \rho_{\text {mass }, 2}$.

Finally, for all simulations, a von Neumann boundary condition and a spatially uniform initial concentration of monomers will be considered. See supplementary material for details of numerical implementation and boundary conditions.

First, we consider a situation where $x<x_{s}$ and model the local pressure by $p=p_{a}+p_{0}$, where $p_{a}=p_{m} \sin \left(\omega t-k_{w} x\right)$ and $k_{w}$ is the wavenumber. The variation in the supersaturation ratio in the acoustic field is determined by defining the quantity $\Delta \overline{Z_{1}}$ as follows:

$$
\Delta \overline{Z_{1}}=\frac{\overline{Z_{1}}}{r_{0}}-1
$$

and subsequently, we can write $r(x, t)=\overline{Z_{1}}=r_{0}\left(1+\Delta \overline{Z_{1}}\right)$, where $r(x, t)$ is the spatially and temporally variable supersaturation ratio.

Figure 4 shows the contour plot of the variation in the concentration of monomers across one wavelength in the preshock region over one period of oscillation. Although the pressure magnitude is relatively large, it has a negligible effect on the spatial distribution of monomers. This result is expected as the pressure diffusion is typically a slow process and may

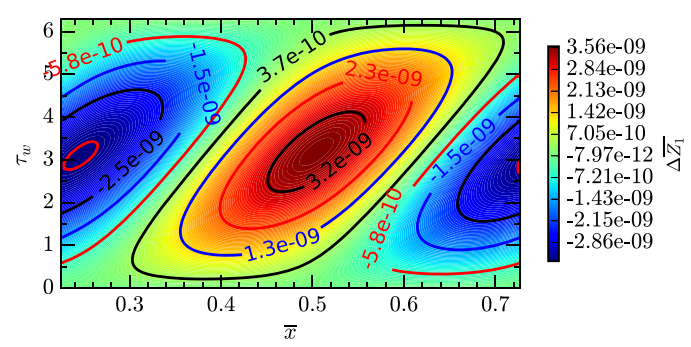

FIG. 4. Change in the concentration of monomers across a wavelength centred about $0.5 x_{s}$ over one period in a traveling wave field with $p_{m}=50 \mathrm{MPa}$ and $f=1 \mathrm{MHz}$. 
become noticeable only over a large time scale or in the presence of a significant pressure gradient. The concentration hot spot (the location where the concentration is maximum) is formed around $\bar{x}=0.48$, at $\tau_{w}=\pi$. The heavier species moves towards the high-pressure region, and consequently, the hot spot smears as the acoustic wave travels.

For a harmonic wave, the gradient and Laplacian of the pressure field are proportional to $k_{w}$ and $k_{w}^{2}$, respectively. This implies that a stronger/weaker effect on the concentration is anticipated at higher/lower excitation frequencies. However, the pressure source terms in the convection-diffusion mass transportation equation are linearly proportional to the pressure magnitude. Consequently, $\Delta \overline{Z_{1}}$ is linearly proportional to the pressure magnitude. More simulations at different $f$ and $p_{m}$ are presented in the supplementary material.

Now, we will consider a shock in $x=2 x_{s}$ at $\tau_{w}=0$. In this region, a full $N$-wave is present and a sharp pressure gradient takes place at the shock location at each time instant. This creates a narrow high-concentration zone right after the shock with a peak of about two orders of magnitude larger than the same wave in the pre-shock zone; see Fig. 5. This increase is followed immediately by a reduction in $\Delta \overline{Z_{1}}$. As time progresses and the shock translates to an adjacent point in space, the concentration rises but it is about two orders of magnitude smaller than the peak and hence less identifiable in the plot. This can be better observed in Fig. 6 which illustrates $\Delta \overline{Z_{1}}$ in the few time instances. In particular, we can see in Fig. 6(b) that $\Delta \overline{Z_{1}}$ settles around about $4 \times 10^{-9}$ which is of the same order of magnitude of its peak in the pre-shock region; see Fig. 4.

A similar narrow variation in the density of medium was also reported for the propagation of weak and strong shock waves in a gaseous medium. ${ }^{58,59}$ They showed that if the sonication carries on for a long time, these narrow high concentration points will disperse. A similar trend is observed here when we run the simulation over a longer time. This

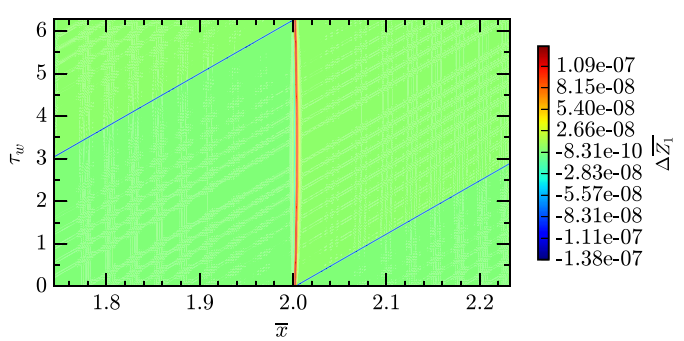

(a)

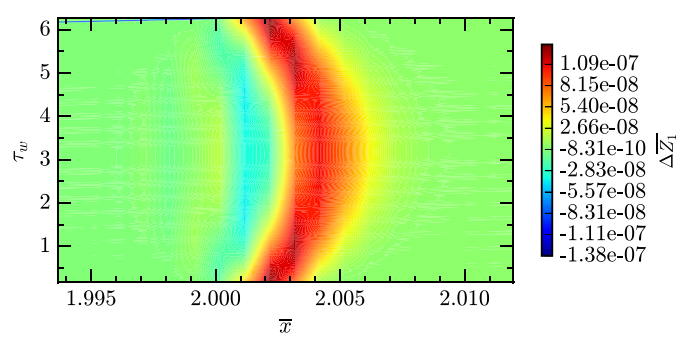

(b)

FIG. 5. (a) Change in the concentration of monomers across a wavelength centred about $2 x_{s}$ over one period in a traveling wave field with $p_{m}=50 \mathrm{MPa}$ and $f=1 \mathrm{MHz}$. (b) Magnified around $2 x_{s}$.

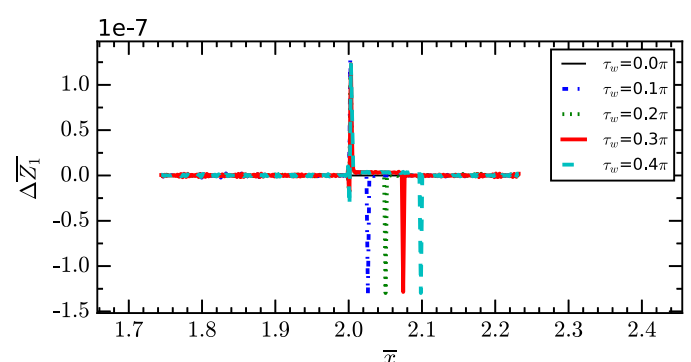

(a)

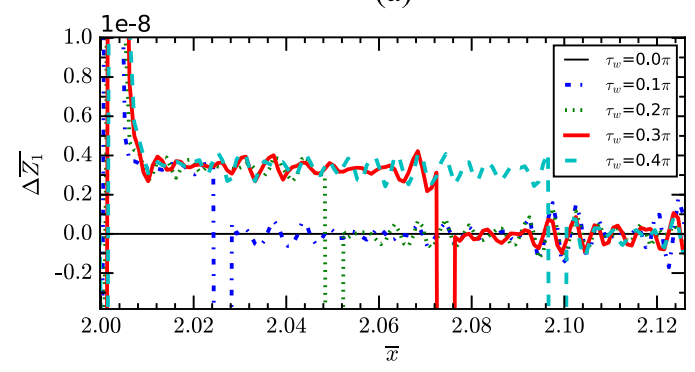

(b)

FIG. 6. (a) Snapshots of the contour plot shown in Fig. 5 in few time instances. (b) Zoomed in around $2 x_{s}$.

is presented in Fig. 7 which shows $\Delta \overline{Z_{1}}$ across a space of about four wavelength long and over ten cycles, i.e., $20 \pi$, with $p_{m}=50 \mathrm{MPa}$ and $f=1 \mathrm{MHz}$. This figure clearly shows that the effect of an acoustic field on the transportation of a typical solute species in a typical aqueous solution (with properties listed in Table II) tends to be negligible. Nevertheless, this effect may become noticeable in the post-shock region and over a large time scale.

The forced mass transportation in this acoustic field is substantially larger (by seven orders of magnitude) than mass transportation by ordinary diffusion. Thus, the effect of ordinary diffusion is negligible and the increased concentration of the solute species is mainly proportional to the gradient of forced mass flux [the second term on the RHS of Eq. (20)]. This can also be deduced from the high value of $P e=1509$ for this driving condition.

If the solute species is made of heavier molecules, a more significant $\Delta \overline{Z_{1}}$ is expected for a given driving pressure. ${ }^{31,34,60}$ Invoking Eq. (33), we can see that the pressure gradient is weighted by $\bar{\beta} / \mathrm{Pe}$ which depends on the solute density and the molecular weight. The latter can take larger values than the former, and its influence on the weight coefficient is perhaps more substantial. To evaluate this effect, we will keep the density constant, i.e., $\rho_{\text {mass }, 2}=2000 \mathrm{~kg} \mathrm{~m}^{-3}$, and choose

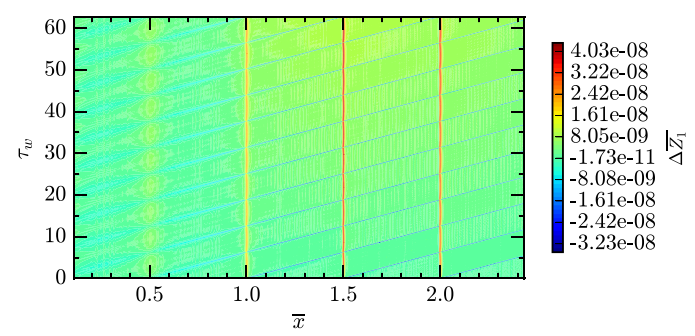

FIG. 7. Change in the concentration of monomers across four wavelengths and over ten periods in a traveling wave field with $p_{m}=50 \mathrm{MPa}$ and $f=1 \mathrm{MHz}$. 


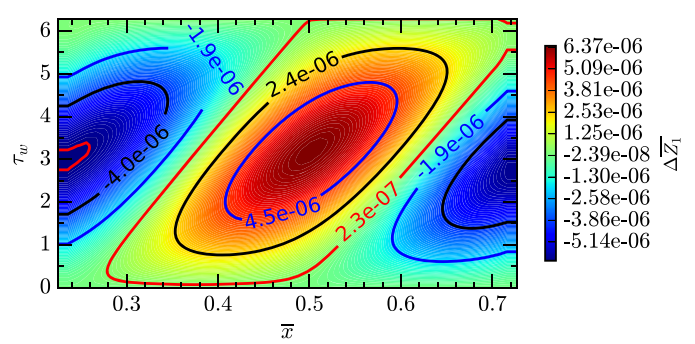

FIG. 8. Change in the concentration of monomers in a pre-shock zone for a solute species with relatively large molecules, $R_{0}=10 \mathrm{~nm}$. The simulation is performed across a wavelength centred about $0.5 x_{s}$ and over one period in a traveling wave field with $p_{m}=50 \mathrm{MPa}$ and $f=1 \mathrm{MHz}$.

a relatively large macromolecule with molecular radius of $R_{0}=10 \mathrm{~nm}$ (this is nearly 44 and 80000 times larger in molecular radius and volume than the ones taken so far). This results in $v_{n}=4000 \mathrm{~nm}^{3}, M_{2}=\mathcal{N}_{\mathcal{A}} v_{n} \rho_{\text {mass }, 2}=5045 \mathrm{~kg} \mathrm{~mol}^{-1}$, and $D=2 \times 10^{-11} \mathrm{~m}^{2} \mathrm{~s}^{-1}$ (calculated by the Stokes-Einstein equation). For the acoustic excitation with $p_{m}=50 \mathrm{MPa}$ and $f=1 \mathrm{MHz}$, we arrive at $\bar{\beta}=-51801$ and $P e=10316$. Consulting the data listed in Table I for the same driving condition, we can see that $\bar{\beta}$ and the Péclet number are increased by 5 orders of magnitude and roughly 1 order of magnitude, respectively, producing a rise in $\bar{\beta} / P e$ by almost 5 orders of magnitude. Figures 8 and 9 depict $\Delta \overline{Z_{1}}$ computed for the solute made of this large molecule in the pre- and post-shock regions. We can observe an enhancement in $\Delta \overline{Z_{1}}$ of the order of nearly 3 at both locations. As before, a very high Péclet number confirms that ordinary diffusion can be overlooked in comparison with the acoustically induced mass transportation.

Now, we need to estimate the effect of variation in the concentration and hence the supersaturation ratio on the thermodynamics of the phase transition. Following our discussion in Sec. II and employing Eq. (34), the contribution of the variable supersaturation ratio to the dimensionless work of the formation of the EDS cluster, i.e., $\Delta \Omega / k_{B} T$, reads

$$
\frac{\Delta \mu}{k_{B} T}=\ln (r)=\ln \left(r_{0}\left(1+\Delta \overline{Z_{1}}\right)\right)=\ln \left(r_{0}\right)+\ln \left(1+\Delta \overline{Z_{1}}\right) \text {. }
$$

As shown above, $\Delta \overline{Z_{1}}$ oscillates around zero and is a relatively small number. Thus, the Taylor series expansion of the second term around zero gives the following first order approximation:

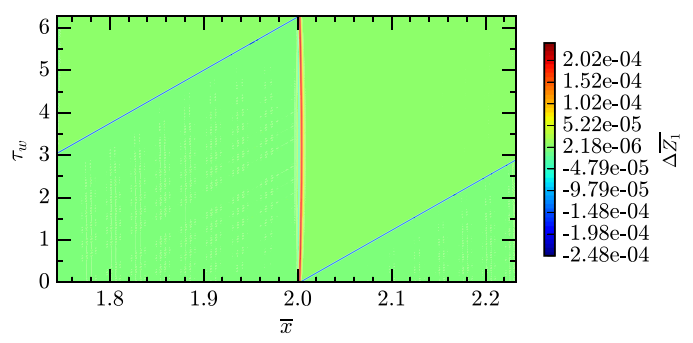

FIG. 9. Change in the concentration of monomers in the post-shock region for a solute species with relatively large molecules, $R_{0}=10 \mathrm{~nm}$. The simulation is performed across a wavelength centred about $2 x_{s}$ and over one period in a traveling wave field with $p_{m}=50 \mathrm{MPa}$ and $f=1 \mathrm{MHz}$.

$$
\frac{\Delta \mu}{k_{B} T} \approx \ln \left(r_{0}\right)+\Delta \overline{Z_{1}}+\mathcal{O}\left(\Delta{\overline{Z_{1}}}^{2}\right)
$$

For example, around the shock region, setting the initial supersaturation ratio $r_{0}=30$ which was used in the previous simulations gives $\Delta \mu / k_{B} T \approx \ln \left(r_{0}\right)+\Delta \overline{Z_{1}}=3.40 \pm 10^{-7}$. In the same situation, the contribution of pressure to the dimensionless work of cluster formation for the same cluster is about $\Delta v \Delta p / k_{B} T=5 \times 10^{-21} / 4 \times 10^{-21} \approx 1.24$. Considering the acoustic field and the bath used in the simulations, the effect of mass transportation on $\Delta \mu$ and consequently the work of cluster formation is of $\mathcal{O}\left(10^{-7}\right)$ and is therefore tentatively negligible, especially in comparison with the pressure effect which is of $\mathcal{O}(1)$.

On the kinetics of cluster formation, the attachment and detachment frequencies are proportional to $r$ and $r^{n_{\sigma^{\prime}}\left(n_{n}\right)}$, respectively; see Eqs. (29) and (31). Given that the variation in $r$ is small, we anticipate an infinitesimal contribution to transition frequencies. For the EDS cluster, $n_{\sigma}{ }^{\prime}\left(n_{n}\right)=0$ and the detachment frequency is predicted to be independent of the supersaturation ratio and mass transportation. To thoroughly investigate this matter, the complete hybrid model in a non-mass conserved system will be solved in Sec. V C for the driving condition in which mass transportation is more noticeable.

\section{Kinetics of sonocrystallisation in a non-mass conserved system}

Preceding results showed that mass transportation may become noticeable at around $\bar{x}=1.99$. Since at this location absorption is the highest, a long excitation will tend to violate the isothermal assumption. Thus, we will solve the cluster dynamics over one wave period. This time is too short for very large clusters to be nucleated, so we can set the largest cluster size to a small value to reduce the number of ordinary differential equations (ODEs) and facilitate the numerical calculations. Here $N=1000$ with $N_{d}=250$ and $a=0.01$ are chosen which gives the largest cluster size of almost 9000 molecules. Cluster transition frequencies are determined using instantaneous supersaturation ratio $r$.

The time-varying supersaturation ratio in a non-mass conserved system in the post-shock region is shown as a contour plot in Fig. 10 and in some spatial points in Fig. 11. The nondimensionalisation coefficients (shown in the supplementary material) are used for numerical calculations. Unlike the preceding simulations, time is scaled by the coefficient of the attachment frequency, i.e., $t_{c}=f_{c}^{-1}$. The results are presented on this time axis instead of $\tau_{w}$.

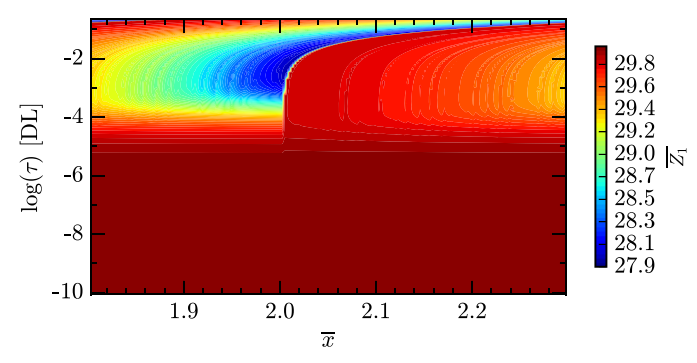

FIG. 10. Concentration of monomers (equivalent to the supersaturation ratio) across a wavelength over time. 


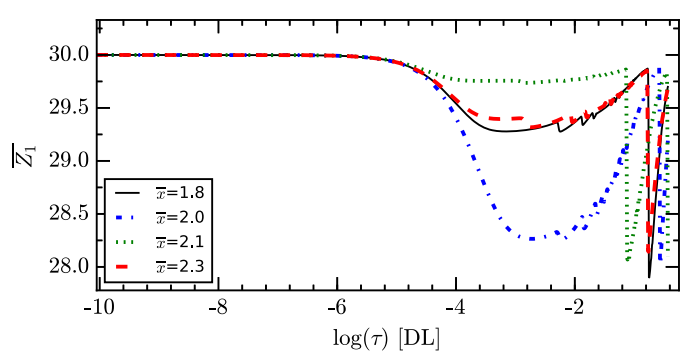

FIG. 11. Concentration of monomers over time at some locations; see Fig. 10.

At locations where initially the pressure amplitude is positive, i.e., points in the rear of the initial location of the shock $\bar{x}<2.1$, nucleation is enhanced which results in a greater reduction of the supersaturation ratio than those locations beyond the shock location. As time progresses, the shock travels in the positive direction of $x$ axis and therefore pressure behind the shock drops. Consequently, the effect of pressure on nucleation at those locations is initially constructive which gradually weakens and becomes repressive when the rarefaction cycle begins.

At the same locations, the concentration of supercritical clusters over time is computed and depicted in Fig. 12. The nucleation time lags $\left(\tau_{n}\right)$ are calculated and shown by the vertical dashed lines. One can see that across a wavelength, they vary by about two orders of magnitude. Given that the time constant is $t_{c}=3.48 \mu \mathrm{s}, \tau_{n}$ changes in the range of nanoseconds to microseconds. This implies that nuclei are created over different time intervals across the wave field, and hence, they will grow to different sizes over the same time. The rate of nucleation is proportional to the inverse of the exponential of the nucleation work which is the lowest just before the shock and the highest just after the shock. Figure 13 displays the spatial distribution of the concentration of supercritical clusters across a wavelength at the end of one period of excitation. Together with Fig. 12, it can be observed that the concentration of supercritical clusters is initially the highest just before the shock (i.e., the blue dashed-dotted curve in Fig. 12) and remains the highest at the end of the sonication period; see Fig. 13. This suggests that during one cycle of excitation, the effect of pressure on the nucleation rate is more significant than the effect of mass transportation.

Figures 14 and 15 show cluster size distributions (CSDs) right before and after the initial location of the shock. The vertical dashed lines display the time-varying size of the critical

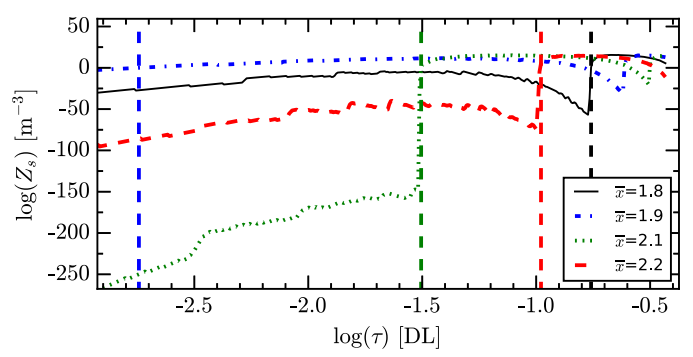

FIG. 12. Concentration of supercritical clusters over time at different locations. Vertical dashed lines indicate the beginning of the nucleation stage at different locations. A similar colour code as the curves is used for these vertical lines.

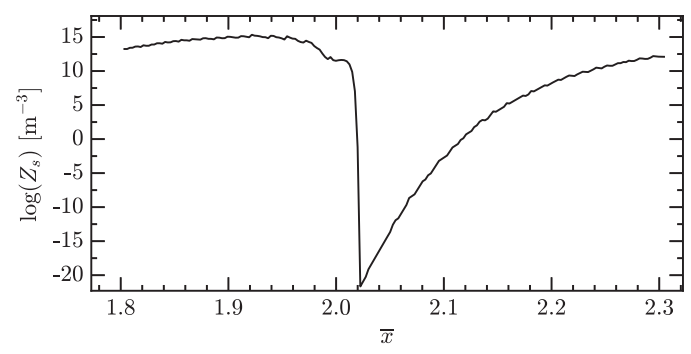

FIG. 13. Concentration of supercritical clusters across space at the end of the first cycle of sonication.

clusters $\left(n^{*}\right)$. Since the simulation time is equal to only one period, these plots only depict the concentration of clusters just in the beginning of nucleation; hence, the growth stage and the Ostwald ripening regime are not observable.

The size of the critical cluster is the extremum of the equilibrium equation which can be found by solving $d \Delta \Omega / d n=0$ for $n$. Thus, differentiating Eq. (6) with respect to $n$ gives

$\frac{\partial \Delta \Omega}{\partial n}=-k_{B} T_{0} \ln (r)\left(1-n_{\sigma}^{\prime}\left(n_{n}\right)\right)+a_{0} \gamma_{\infty} \frac{2}{3} n_{n}^{-1 / 3} n_{n}^{\prime}\left(n_{n}\right) \Delta n_{e x c}^{\prime} v \Delta p$,

which has the following solution for an EDS nucleus:

$$
n_{e}^{*}=\left(\frac{2}{3} \frac{\frac{a_{0} \gamma_{\infty}}{k_{B} T_{0}}}{\ln (r)+\frac{k_{\rho} v \Delta p}{k_{B} T_{0}}}\right)^{3} .
$$

For a non-EDS nucleus, the solution of Eq. (37) ought to be found numerically. Here, the supersaturation ratio is denoted by $r$ instead of $r_{0}$ as it refers to the time-varying supersaturation and includes the effect of mass transportation; see Eq. (35). In both EDS and non-EDS clusters, we can see that the dependence of $n^{*}$ on mass transportation and the pressure amplitude are given by $\Delta \overline{Z_{1}}$ [of first order accuracy by using Eq. (36)] and $k_{\rho} v \Delta p / k_{B} T_{0}$, respectively. Taking the range of $\Delta \overline{Z_{1}}$ into account and given that the smallest size of $n^{*}$ at both locations are equal, we may deduce that the effect of mass transportation on the size of nuclei is negligible compared to the pressure effect [the former is of $\mathcal{O}\left(10^{-7}\right)$ whereas the latter is of $\mathcal{O}(1)$ with the value of 1.24]. Consequently, we may extend this conclusion and correlate the spatial variation of the CSD to mainly the pressure field.

We have studied the effect of pressure magnitude and mass transportation on the nucleation work under an isothermal condition. It was shown that for the considered pressure

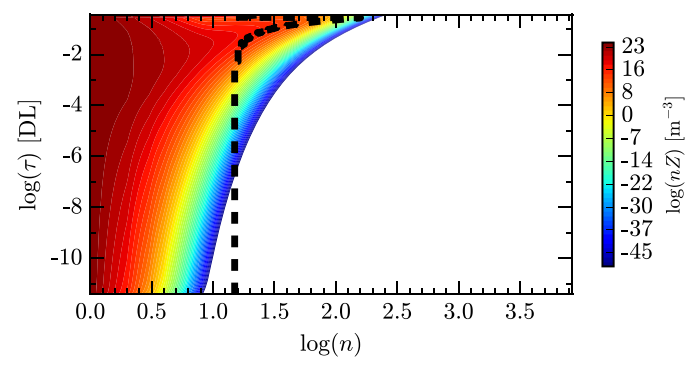

FIG. 14. CSD at $\bar{x}=1.99$. Vertical dashed line shows the size of the critical cluster. 


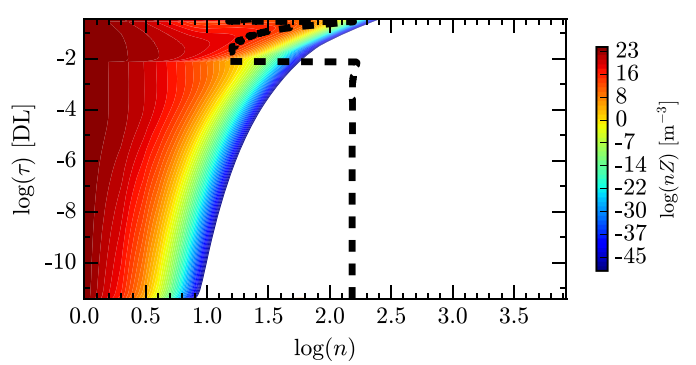

FIG. 15. CSD at $\bar{x}=2.02$. Vertical dashed line shows the size of the critical cluster.

field, the effect of mass transportation was negligible compared to the pressure effect. Now, we are interested to evaluate their effect on the excess free energy. In the case of the conventional form of the classical nucleation theory (CNT), the excess free energy reads $F_{\sigma, c l}=a_{0} \gamma_{\infty} n^{2 / 3}$ and only varies with size when the temperature is constant. Thus, the excess free energy modeled in the conventional form of the CNT is independent of both time-varying supersaturation ratio and pressure amplitude. Considering isothermal wave propagation in an incompressible solution, the excess free energy can be obtained by manipulating Eq. (6). Using $\Delta n_{e x c}=k_{\rho} n_{n}+n_{\sigma}$ and $n=n_{n}+n_{\sigma}$, Eq. (6) re-arranges to

$$
\begin{aligned}
\Delta \Omega\left(n_{n}, n_{\sigma}, p\right)= & -n\left(k_{B} T_{0} \ln (r)+k_{\rho} v \Delta p\right) \\
& +n_{\sigma}\left(k_{B} T_{0} \ln (r)+k_{\rho} v \Delta p\right)-n_{\sigma} v \Delta p \\
& +a_{0} \gamma_{\infty} n_{n}^{2 / 3} .
\end{aligned}
$$

The terms inside the brackets are the instantaneous difference in chemical potentials in the wave field, i.e., $\Delta \mu(r, p)$, accounting for the effect of the instantaneous supersaturation ratio $(r)$ and pressure amplitude $(p)$ on chemical potentials. Comparing Eq. (39) with Eq. (4), we can deduce the excess free energy of cluster formation which entails pressure and mass transportation effects as follows:

$$
F_{\sigma, 1}(r, p)=n_{\sigma}\left(k_{B} T_{0} \ln (r)+k_{\rho} v \Delta p\right)-n_{\sigma} v \Delta p+a_{0} \gamma_{\infty} n_{n}^{2 / 3} .
$$

This equation was used to determine the excess free energy for the simulation condition explained above, i.e., across a wavelength in a post-shock region with the driving condition of $p_{m}=50 \mathrm{MPa}$ and $f=1 \mathrm{MHz}$ (see Figs. 10-15). The excess free energy of a cluster of size $n=10$ (see the supplementary material for $n=1000$ ) identified with $\lambda=0.35$ is depicted in Fig. 16. For this supersaturated solution (with

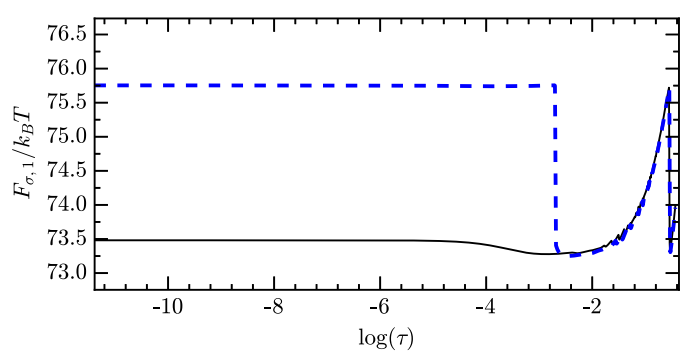

FIG. 16. Excess free energy of a cluster of size $n=10$ over time at two locations; just before the shock $\bar{x}=1.99$ (the black solid curve) and just after the shock $\bar{x}=2.01$ (the blue dashed curve). $F_{\sigma, 1}$ is calculated by Eq. (40).

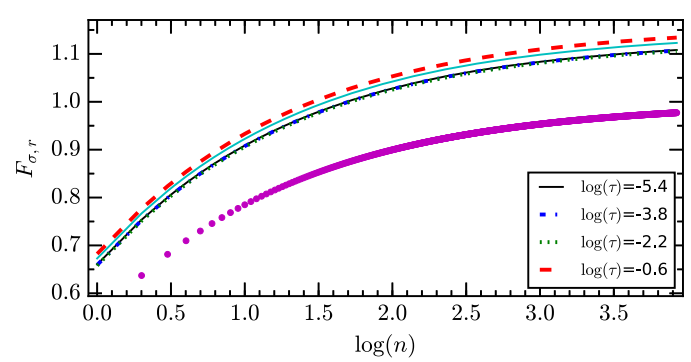

FIG. 17. The ratio of the excess free energy for the non-EDS cluster with $\lambda=0.35$ to the excess free energy of the EDS cluster of the same size $\left(F_{\sigma, c l}\right)$, calculated at $\bar{x}=1.99$. Curves present readings at different time instants shown in the legend. The dotted curve in magenta is the ratio of the contribution of the surface tension term and the cyan solid curve is $F_{\sigma, 1}$ with a constant supersaturation ratio in the absence of pressure; see the text for details.

$\left.k_{\rho}>0\right)$ and acoustic parameters, the following points can be deduced from this result: (i) the excess free energy fluctuates out of phase with pressure, (ii) the effect of mass transportation on $F_{\sigma, 1}$ is negligible as we could not identify a noticeable difference in the extremum values at different locations, and (iii) $F_{\sigma, 1}$ becomes a minimum behind the shock and a maximum right after the shock.

To further elaborate on these results and the effect of $\lambda$, the ratio of the excess free energy of these non-EDS clusters calculated by Eq. (40) at location $\bar{x}=1.99$ to the excess free energy of the EDS clusters of the identical size, i.e., $F_{\sigma, c l}$, is calculated and exhibited in Fig. 17. The magenta dotted curve in this figure compares the contribution of the surface tension term to the excess free energy in the non-EDS cluster relative to the classical cluster, i.e., $a_{0} \gamma_{\infty} n_{n}^{2 / 3} / F_{\sigma, c l}$. The cyan solid curve displays the excess free energy calculated at the constant initial supersaturation ratio of $r_{0}=30$ in the absence of pressure, i.e., $F_{\sigma, 1}=n_{\sigma} k_{B} T_{0} \ln \left(r_{0}\right)+a_{0} \gamma_{\infty} n_{n}^{2 / 3}$, and hence does not account for variation in the supersaturation ratio due to mass transportation and nucleation, as well as the pressure effect. The other curves show dynamic excess free energy with time-varying supersaturation ratio and pressure amplitude at different time instants, i.e., $F_{\sigma, 1}$ given by Eq. (40). This figure shows that for this solution and acoustic field: (i) the dynamic excess free energy, i.e., $F_{\sigma, 1}(r, p)$, fluctuates around $F_{\sigma, 1}\left(r_{0}\right)$ and (ii) the contribution of mass transportation and the pressure magnitude to the excess free energy of clusters is less pronounced than the supersaturation ratio itself (the amplitude of this oscillation is fairly small). Furthermore, we can see that the excess free energy of non-EDS clusters is substantially greater than the excess free energy of classical clusters for these parameters, i.e., $k_{\rho}>0$ and $\lambda=0.35$. It is shown by Haqshenas et al. ${ }^{12}$ that the effective surface tension based on the nonEDS cluster model is determined by $\gamma_{\mathrm{eff}}=\gamma_{\infty} F_{\sigma, r}$, where $F_{\sigma, r}=F_{\sigma, 1} / F_{\sigma, c l}$. Therefore, scaling the curves shown in Fig. 17 by $\gamma_{\infty}$ (evaluated at the initial equilibrium state) would give us an estimation of the size-dependent and time-varying surface tension including the effects of dynamic pressure and supersaturation ratio.

\section{CONCLUSION}

We studied the thermodynamics and kinetics of sonocrystallisation in a non-mass conserved system. This work was the 
extension of our previous paper. ${ }^{12}$ Here, we formulated the evolution of cluster concentration over time in a non-mass conserved system by including mass transport in the hybrid kinetics model; see Eqs. (16), (23), and (24). In the limit of a dilute solution where we can reasonably assume that only monomers transport through the system, the hybrid model simplifies to Eq. (25) which transforms into Eqs. (26) and (27) for incompressible solutions with and without the Ostwald ripening process, respectively. These equations together with equations derived for cluster transition frequencies in an acoustic field [see the work of Haqshenas et al. ${ }^{12}$ for generic formulas or Eqs. (28) and (31) for an isothermal condition] allow us to model the effects of acoustic waves on the aggregative and non-aggregative mechanisms of the kinetics of sonocrystallisation.

Utilising this model under an isothermal condition in a non-mass conserved system, we observed that the boundary conditions, the driving parameters of the acoustic field, and the spatial location of the system (i.e., at a pre- or post-shock location) are important and can influence the thermodynamics and kinetics of cluster formation. However, in the present case, the effect of the pressure field is more significant on the thermodynamics of phase transition than the mass transportation. Therefore, acoustic waves influence nucleation and growth through thermodynamic effects associated with pressure fluctuations (the aggregative mechanism in the kinetics model) rather than hydrodynamic effects. Nonetheless, this conclusion should be tempered as mass transportation may vary when the boundary conditions change or the acoustic streaming is accounted for in the mass transportation equation, e.g., in the near-field of an oscillating bubble ${ }^{34}$ or close to a hard wall in a confined system. ${ }^{61}$ This highlights the importance of the profound and accurate characterisation of the system in experiments which is usually overlooked in the sonocrystallisation literature.

Simulations with a solute of different molecular weight showed that the higher the molecular weight of the solute species, the more considerable is the effect of mass transportation. Nevertheless, this results in a lower mobility of monomers which reduces the attachment frequency and consequently may weaken the effect of mass transportation on the kinetics of nucleation. These results suggest that sonocrystallisation can be more effective for producing solid particles with higher molecular weights.

The excess free energy of cluster formation in an acoustic field was also calculated using the non-EDS cluster model developed in our previous work. ${ }^{12}$ It was shown that both the pressure magnitude and the supersaturation ratio influence the excess free energy, and it is noticeably different from the predictions of the conventional form of the CNT. Since the acoustic field and the supersaturation ratio vary spatially, the excess free energy of cluster formation, likewise the clustering work, will vary spatially in an acoustic field.

Although we used a one-dimensional acoustic field for numerical simulations, the model is generic and can be used to determine the thermodynamics and kinetics of cluster formation for the EDS or non-EDS clusters in an arbitrary acoustic field. Simulations presented in this work based on this simple one-dimensional acoustic wave model showed that the contribution of the non-aggregative mechanism depends on the magnitude and wavelength of acoustic waves and generally tends to be negligible unless shock waves are present or a special boundary condition is imposed. Studying the effect of acoustic streaming and different boundary conditions on the kinetics of sonocrystallisation will be the subject of the forthcoming paper.

\section{SUPPLEMENTARY MATERIAL}

See supplementary material for the details of the nondimensionalisation of the master equation and numerical implementation. Additional simulation results are presented as well.

\section{ACKNOWLEDGMENTS}

This work was supported by the EPSRC (Grant No. $\mathrm{EP} / \mathrm{I} 031480 / 1)$.

\section{APPENDIX A: MONOMERS MASS FLUX}

Considering only monomers transport in the solution, the solution can be considered as a binary mixture. The molecular mass flux for an ideal binary solution in the absence of external forces becomes ${ }^{31}$

$$
\begin{aligned}
\mathbf{j}_{[1]}= & -\frac{C_{m}^{2}}{\rho_{m}} M_{1} M_{2} D \nabla x_{2}-D_{2}^{T} \nabla \ln T \\
& -\frac{C_{m}^{2}}{\rho_{m} \mathcal{R} T} M_{2}^{2} M_{1} D x_{2}\left(\frac{\overline{V_{2}}}{M_{2}}-\frac{1}{\rho_{m}}\right) \nabla p,
\end{aligned}
$$

where $\mathbf{j}_{[1]}$ is the molecular mass flux of monomers, $C_{m}=C_{1}$ $+C_{2}=\rho_{m} / M_{m}$ is the total molar concentration of the solution (the molar density of the solution), $D, x_{2}$, and $D_{2}^{T}$ are the diffusivity, the mole fraction, and the thermal diffusion coefficient of the solute in the solution, respectively. $\overline{V_{2}}=M_{2} / \rho_{2}$ is the partial molal volume, and $M_{m}$ and $M_{1}$ are the molar mass of the solution and solvent, respectively. Approximating the mole fraction by the number concentration ratio of monomers gives $x_{2}=Z_{1}^{m t} / C_{m} \mathcal{N}_{\mathcal{A}}$, and substituting this in the above equation results in Eq. (18).

\section{APPENDIX B: NON-MASS CONSERVED FORM OF THE FPE}

Equation (23) shows the Szilard equation in a nonmass conserved system. This equation is reproduced in the following:

$$
\begin{aligned}
\frac{\partial}{\partial t} Z_{n}= & f_{n-1}(t) Z_{n-1}-g_{n}(t) Z_{n}-f_{n}(t) Z_{n}+g_{n+1}(t) Z_{n+1} \\
& -Z_{n} \nabla \cdot \mathbf{u}-\mathbf{u} \cdot \nabla Z_{n}-\frac{\mathcal{N}_{\mathcal{A}}}{M_{2} n} \nabla \cdot \mathbf{j}_{[n]} .
\end{aligned}
$$

Following the work of Kashchiev, ${ }^{13}$ we can derive the continuous format of this equation by writing the truncated Taylor series expansion of $f_{n-1}(t) Z_{n-1}$ and $g_{n+1}(t) Z_{n+1}$ terms about point $n$, 


$$
\begin{aligned}
f(n-1, t) Z(n-1, t)= & f(n, t) Z(n, t) \\
& +\frac{\partial}{\partial n}[f(n, t) Z(n, t)](n-1-n) \\
& +\frac{1}{2} \frac{\partial^{2}}{\partial n^{2}}[f(n, t) Z(n, t)](n-1-n)^{2}
\end{aligned}
$$

and

$$
\begin{aligned}
g(n+1, t) Z(n+1, t)= & g(n, t) Z(n, t) \\
& +\frac{\partial}{\partial n}[g(n, t) Z(n, t)](n+1-n) \\
& +\frac{1}{2} \frac{\partial^{2}}{\partial n^{2}}[g(n, t) Z(n, t)](n+1-n)^{2}
\end{aligned}
$$

Inserting these two equations into Eq. (B1) yields

$$
\begin{aligned}
\frac{\partial}{\partial t} Z(n)= & -\frac{\partial}{\partial n}((f(n, t)-g(n, t)) Z(n) \\
& \left.-\frac{1}{2} \frac{\partial[(f(n, t)+g(n, t)) Z(n)]}{\partial n}\right)-Z(n) \nabla \cdot \mathbf{u} \\
& -\mathbf{u} \cdot \nabla Z(n)-\frac{\mathcal{N}_{\mathcal{A}}}{M_{2} n}\left(\nabla \cdot \mathbf{j}_{[n]}\right)
\end{aligned}
$$

and employing the definitions of the dispersion and growth rates, Eq. (9), simplifies this equation to the following format:

$$
\begin{aligned}
\frac{\partial}{\partial t} Z(n)= & -\frac{\partial}{\partial n}\left(v(n) Z(n)-\frac{1}{2} \frac{\partial[d(n, t) Z(n)]}{\partial n}\right)-Z(n) \nabla \cdot \mathbf{u} \\
& -\mathbf{u} \cdot \nabla Z(n)-\frac{\mathcal{N}_{\mathcal{A}}}{M_{2} n}\left(\nabla \cdot \mathbf{j}_{[\mathbf{n}]}\right) .
\end{aligned}
$$

This is the non-mass conserved form of the FPE.

\section{APPENDIX C: MATERIAL PROPERTIES}

The physiochemical properties of a generic aqueous solution at room temperature $(T=293 \mathrm{~K})$ and atmospheric pressure were used in the simulations of crystal nucleation. These properties are taken from Table 6.1 in the work of Kashchiev. ${ }^{13}$ Following this reference, we consider the new phase to be denser than the old phase with a typical value of $\Delta v=10^{-28} \mathrm{~m}^{3}$. This gives $k_{\rho}=0.66$. All these parameters are summarised in Table II.

Additionally, following Ref. 34, we choose the average value of $\rho_{\text {mass }, 2}=2000 \mathrm{~kg} \mathrm{~m}^{-3}$ for the mass density of the solute species and calculate $M_{2}$ by $M_{2}=\mathcal{N}_{\mathcal{A}} v_{n} \rho_{\text {mass }, 2}=0.06 \mathrm{~kg} \mathrm{~mol}^{-1}$.

For simulations including pressure variations, the reference state is considered to be at room temperature and

TABLE II. Solution properties at $T=293 \mathrm{~K}$ and atmospheric pressure.

\begin{tabular}{lccc}
\hline \hline Parameter & Value & Parameter & Value \\
\hline$v_{n}\left(\mathrm{~nm}^{3}\right)$ & 0.05 & $v\left(\mathrm{~nm}^{3}\right)$ & 0.15 \\
$\rho_{n}\left(\mathrm{~nm}^{-3}\right)$ & 20.0 & $\rho\left(\mathrm{nm}^{-3}\right)$ & 6.67 \\
$k_{\rho}(\mathrm{DL})$ & 0.66 & $R_{0}(\mathrm{~nm})$ & 0.23 \\
$C_{e}\left(\mathrm{~m}^{-3}\right)$ & $10^{23}$ & $\gamma\left(\mathrm{mJ} \mathrm{m}^{-2}\right)$ & 100 \\
$D\left(\mathrm{~m}^{2} \mathrm{~s}^{-1}\right)$ & $10^{-9}$ & & \\
\hline
\end{tabular}

TABLE III. Water properties at $T=293 \mathrm{~K}$ and atmospheric pressure.

\begin{tabular}{lc}
\hline \multicolumn{1}{c}{ Parameter } & Value \\
\hline$M\left(\mathrm{H}_{2} \mathrm{O}\right)\left(\mathrm{kg} \mathrm{mol}^{-1}\right)$ & $0.018015^{\mathrm{a}}$ \\
$\rho\left(\mathrm{H}_{2} \mathrm{O}\right)\left(\mathrm{kg} \mathrm{m}^{-3}\right)$ & $998.24^{\mathrm{a}}$ \\
$\beta(\mathrm{DL})$ & $3.5^{\mathrm{a}}$ \\
$\eta_{B}(\mathrm{~Pa} \mathrm{~s})$ & $2.4 \times 10^{-3 \mathrm{~b}}$ \\
$\eta(\mathrm{Pa} \mathrm{s})$ & $1.0020 \times 10^{-3 \mathrm{c}}$ \\
$c_{0}\left(\mathrm{~m} \mathrm{~s}^{-1}\right)$ & $1482.2^{\mathrm{c}}$ \\
$C_{p}\left(\mathrm{~J} \mathrm{~K}^{-1}\right)$ & $75.377^{\mathrm{c}}$ \\
$\kappa\left(\mathrm{W} \mathrm{m}^{-1} \mathrm{~K}^{-1}\right)$ & $0.598^{\mathrm{c}}$ \\
\hline \hline
\end{tabular}

${ }^{\mathrm{a}}$ From Ref. 8.

${ }^{b}$ From Ref. 62.

${ }^{\mathrm{c}}$ From Ref. 63.

atmospheric pressure, $T_{0}=293 \mathrm{~K}$ and $p_{0}=0.1 \mathrm{MPa}$, respectively. Therefore, the values reported in this table refer to the physicochemical properties at the reference state.

Burgers' and mass transportation equations are solved under isothermal condition at $T=293 \mathrm{~K}$. The properties of water are given in Table III.

${ }^{1}$ L. A. Chambers, "The emission of visible light from cavitated liquids," J. Chem. Phys. 5, 290-292 (1937).

${ }^{2}$ K. Davitt, E. Rolley, F. Caupin, A. Arvengas, and S. Balibar, "Equation of state of water under negative pressure," J. Chem. Phys. 133, 174507 (2010).

${ }^{3}$ V. G. Baidakov, "Spontaneous cavitation in a Lennard-Jones liquid: Molecular dynamics simulation and the van der Waals-Cahn-Hilliard gradient theory," J. Chem. Phys. 144, 074502 (2016).

${ }^{4}$ G. Ruecroft, D. Hipkiss, T. Ly, N. Maxted, and P. Cains, "Sonocrystallization: The use of ultrasound for improved industrial crystallization," Org. Process Res. Dev. 9, 923-932 (2005).

${ }^{5}$ T. A. Litovitz, E. H. Carnevale, and P. A. Kendall, "Effects of pressure on ultrasonic relaxation in liquids," J. Chem. Phys. 26, 465-468 (1957).

${ }^{6} \mathrm{R}$. Kono and T. Yamaoka, "Concentration fluctuation process in ethanolwater mixture," J. Chem. Phys. 68, 5206-5213 (1978).

${ }^{7}$ L. E. Kinsler, A. R. Frey, A. B. Coppens, and J. V. Sanders, in Fundamentals of Acoustics, 4th ed., by L. E. Kinsler, A. R. Frey, A. B. Coppens, and J. V. Sanders, (Wiley-VCH, 1999), Vol. 1, p. 560, ISBN: 0-471-84789-5.

${ }^{8} \mathrm{M}$. Hamilton and D. Blackstock, Nonlinear Acoustics (Academic Press, 1998).

${ }^{9}$ V. Griffing and D. Sette, "Luminescence produced as a result of intense ultrasonic waves," J. Chem. Phys. 23, 503-509 (1955).

${ }^{10}$ E. B. Flint and K. S. Suslick, "The temperature of cavitation," Science 253, 1397-1399 (1991).

${ }^{11}$ K. S. Suslick and D. J. Flannigan, "Inside a collapsing bubble: Sonoluminescence and the conditions during cavitation," Annu. Rev. Phys. Chem. 59, 659-683 (2008).

${ }^{12}$ S. R. Haqshenas, I. J. Ford, and N. Saffari, "Modelling the effect of acoustic waves on nucleation," J. Chem. Phys. 145, 024315 (2016).

${ }^{13}$ D. Kashchiev, Nucleation (Butterworth-Heinemann, 2000).

${ }^{14} \mathrm{G}$. Zinsmeister, "Theory of thin film condensation. Part B: Solution of the simplified condensation equation," Thin Solid Films 2, 497-507 (1968).

${ }^{15} \mathrm{G}$. Zinsmeister, "Theory of thin film condensation. Part C: Aggregate size distribution in island films," Thin Solid Films 4, 363-386 (1969).

${ }^{16}$ M. Zinke-Allmang, L. C. Feldman, and M. H. Grabow, "Clustering on surfaces,” Surf. Sci. Rep. 16, 377-463 (1992).

${ }^{17}$ J. Venables, G. Spiller, and M. Hanbucken, "Nucleation and growth of thin films," Rep. Prog. Phys. 47, 399 (1984).

${ }^{18}$ J. Nieminen and K. Kaski, "Rate-equation study of nucleation and growth of thin films. I. growth of one monolayer," Phys. Rev. A 40, 2088 (1989).

${ }^{19} \mathrm{C}$. Ratsch and J. Venables, "Nucleation theory and the early stages of thin film growth," J. Vac. Sci. Technol., A 21, S96-S109 (2003).

${ }^{20}$ Y. Han, E. Gaudry, T. J. Oliveira, and J. W. Evans, "Point island models for nucleation and growth of supported nanoclusters during surface deposition," J. Chem. Phys. 145, 211904 (2016).

${ }^{21} \mathrm{P}$. G. Hill, "Condensation of water vapour during supersonic expansion in nozzles," J. Fluid Mech. 25, 593-620 (1966). 
${ }^{22}$ J. M. Deutch and B. U. Felderhof, "Hydrodynamic effect in diffusioncontrolled reaction," J. Chem. Phys. 59, 1669-1671 (1973).

${ }^{23} \mathrm{~F}$. Bakhtar and M. M. Tochai, "An investigation of two-dimensional flows of nucleating and wet steam by the time-marching method," Int. J. Heat Fluid Flow 2, 5-18 (1980).

${ }^{24}$ S. Melis, M. Verduyn, G. Storti, M. Morbidelli, and J. Bałdyga, "Effect of fluid motion on the aggregation of small particles subject to interaction forces," AIChE J. 45, 1383-1393 (1999).

${ }^{25} \mathrm{~J}$. Badyga and W. Orciuch, "Some hydrodynamic aspects of precipitation," Powder Technol. 121, 9-19 (2001).

${ }^{26}$ D. Mitrakos, E. Hinis, and C. Housiadas, "Sectional modeling of aerosol dynamics in multi-dimensional flows," Aerosol Sci. Technol. 41, 10761088 (2007).

${ }^{27}$ N. D. Pasquale, D. Marchisio, P. Carbone, and A. Barresi, "Identification of nucleation rate parameters with MD and validation of the CFD model for polymer particle precipitation," Chem. Eng. Res. Des. 91, 2275-2290 (2013).

28 J. K. Baird and L. Guo, "Free convection and surface kinetics in crystal growth from solution," J. Chem. Phys. 109, 2503-2508 (1998).

${ }^{29}$ V. V. Pulikkottil and R. I. Sujith, "Instability mechanisms in a lowMach-number reacting flow from coupled convection-reaction-diffusion equations," Phys. Fluids 27, 074101 (2015).

${ }^{30}$ F. T. Ferguson, R. H. Heist, and J. A. Nuth, "The influence of buoyant convection on the nucleation of n-propanol in thermal diffusion cloud chambers," J. Chem. Phys. 132, 204510 (2010).

${ }^{31}$ R. B. Bird, W. E. Stewart, and E. N. Lightfoot, Transport Phenomena, Wiley International Edition (Wiley, 1960).

${ }^{32}$ U. Marconi and S. Melchionna, "Multicomponent diffusion in nanosystems," J. Chem. Phys. 135, 044104 (2011).

${ }^{33}$ W. J. Archibald, "The process of diffusion in a centrifugal field of force," Phys.Rev. 53, 746 (1938).

${ }^{34}$ O. Louisnard, F. J. Gomez, and R. Grossier, "Segregation of a liquid mixture by a radially oscillating bubble," J. Fluid Mech. 577, 385-415 (2007).

${ }^{35} \mathrm{~S}$. Newman and F. Eirich, "Particle shape and the concentration dependence of sedimentation and diffusion," J. Colloid Sci. 5, 541-549 (1950).

${ }^{36} \mathrm{~S}$. Winkelmann and C. Schütte, "The spatiotemporal master equation: Approximation of reaction-diffusion dynamics via Markov state modeling," J. Chem. Phys. 145, 214107 (2016).

${ }^{37}$ L. V. King, "On the acoustic radiation pressure on spheres," Proc. R. Soc. A 147, 212-240 (1934).

${ }^{38}$ R. Löfstedt and S. Putterman, "Theory of long wavelength acoustic radiation pressure,” J. Acoust. Soc. Am. 90, 2027-2033 (1991).

${ }^{39} \mathrm{M}$. Settnes and H. Bruus, "Forces acting on a small particle in an acoustical field in a viscous fluid," Phys. Rev. E 85, 016327 (2012).

${ }^{40}$ H. Bruus, J. Dual, J. Hawkes, M. Hill, T. Laurell, J. Nilsson, S. Radel, S. Sadhal, and M. Wiklund, "Forthcoming lab on a chip tutorial series on acoustofluidics: Acoustofluidics-exploiting ultrasonic standing wave forces and acoustic streaming in microfluidic systems for cell and particle manipulation," Lab Chip 11, 3579-3580 (2011).

${ }^{41}$ A. Eller and H. G. Flynn, "Rectified diffusion during nonlinear pulsations of cavitation bubbles," J. Acoust. Soc. Am. 37, 493-503 (1965).

${ }^{42} \mathrm{~S}$. Fujikawa and T. Akamatsu, "Effects of the non-equilibrium condensation of vapour on the pressure wave produced by the collapse of a bubble in a liquid," J. Fluid Mech. 97, 481-512 (1980).

${ }^{43}$ M. M. Fyrillas and A. J. Szeri, "Dissolution or growth of soluble spherical oscillating bubbles," J. Fluid Mech. 277, 381-407 (1994).
${ }^{44}$ B. D. Storey and A. J. Szeri, "Water vapour, sonoluminescence and sonochemistry," Proc. R. Soc. A 456, 1685-1709 (2000).

${ }^{45}$ B. D. Storey and A. J. Szeri, "Mixture segregation within sonoluminescence bubbles," J. Fluid Mech. 396, 203-221 (1999).

${ }^{46}$ T. Vetter, M. Iggland, D. R. Ochsenbein, F. S. Hanseler, and M. Mazzotti, "Modeling nucleation, growth, and Ostwald ripening in crystallization processes: A comparison between population balance and kinetic rate equation," Cryst. Growth Des. 13, 4890-4905 (2013).

${ }^{47}$ G. Ozkan and P. Ortoleva, "A mesoscopic model of nucleation and Ostwald ripening/stepping: Application to the silica polymorph system," J. Chem. Phys. 112, 10510-10525 (2000).

${ }^{48} \mathrm{Z}$. Kozísek and P. Demo, "Influence of initial conditions on homogeneous nucleation kinetics in a closed system," J. Chem. Phys. 123, 144502 (2005).

${ }^{49}$ S. Shaw and P. Spelt, "Shock emission from collapsing gas bubbles," J. Fluid Mech. 646, 363-373 (2010).

${ }^{50}$ C. Cogné, S. Labouret, R. Peczalski, O. Louisnard, F. Baillon, and F. Espitalier, "Theoretical model of ice nucleation induced by acoustic cavitation. Part 1: Pressure and temperature profiles around a single bubble," Ultrason. Sonochem. 29, 447 (2016).

${ }^{51}$ C. D. Ohl, T. Kurz, R. Geisler, O. Lindau, and W. Lauterborn, "Bubble dynamics, shock waves and sonoluminescence," Philos. Trans. R. Soc., A 357, 269-294 (1999).

${ }^{52}$ I. Akhatov, O. Lindau, A. Topolnikov, R. Mettin, N. Vakhitova, and W. Lauterborn, "Collapse and rebound of a laser induced cavitation bubble," Phys. Fluids 13, 2805-2819 (2001).

${ }^{53}$ J. K. Platten, "The Soret effect: A review of recent experimental results," J. Appl. Mech. 73, 5-15 (2006).

${ }^{54} \mathrm{~A}$. Prosperetti, "Nonlinear oscillations of gas bubbles in liquids. Transient solutions and the connection between subharmonic signal and cavitation," J. Acoust. Soc. Am. 57, 810-821 (1975).

${ }^{55}$ D. Kashchiev, "Nucleation at variable supersaturation," Surf. Sci. 18, $293-$ 297 (1969).

${ }^{56} \mathrm{H}$. Mitome, "An exact solution for finite-amplitude plane sound waves in a dissipative fluid,” J. Acoust. Soc. Am. 86, 2334-2338 (1989).

${ }^{57}$ S. R. Haqshenas, "Modelling the effect of acoustic waves on the thermodynamics and kinetics of crystal nucleation from a solution," Ph.D. thesis, University College London, 2017.

${ }^{58} \mathrm{~T}$. Yano and Y. Inoue, "Quasisteady streaming with rarefaction effect induced by asymmetric sawtooth-like plane waves," Phys. Fluids 8, 25372551 (1996).

${ }^{59} \mathrm{~T}$. Yano, "Mass, momentum and total excess energy transported by a weak planen wave," Shock Waves 6, 313-322 (1996).

${ }^{60} \mathrm{~J}$. Vrentas and C. Vrentas, Diffusion and Mass Transfer (CRC Press, 2016).

${ }^{61}$ B. D. Goddard, A. Nold, and S. Kalliadasis, "Dynamical density functional theory with hydrodynamic interactions in confined geometries," J. Chem. Phys. 145, 214106 (2016).

${ }^{62}$ X. He, H. Wei, J. Shi, J. Liu, S. Li, W. Chen, and X. Mo, "Experimental measurement of bulk viscosity of water based on stimulated Brillouin scattering," Opt. Commun. 285, 4120-4124 (2012).

${ }^{63}$ E. W. Lemmon, M. O. McLinden, and D. G. Friend, "Thermophysical properties of fluid systems," in NIST Chemistry WebBook, NIST Standard Reference Database Number 69, edited by P. J. Linstrom and W. G. Mallard (National Institute of Standards and Technology, Gaithersburg, MD, 2016), p. 20899. 